\title{
COS-burst: Observations of the Impact of Starburst-driven Winds on the Properties of the Circum-galactic Medium
}

\author{
Timothy Heckman $^{1}$ (D), Sanchayeeta Borthakur ${ }^{1}$ (D), Vivienne Wild ${ }^{2}$, David Schiminovich ${ }^{3}$, and Rongmon Bordoloi ${ }^{4,5}$ \\ ${ }^{1}$ Center for Astrophysical Sciences, Department of Physics \& Astronomy, Johns Hopkins University, Baltimore, MD 21218, USA; theckma1@jhu.edu \\ ${ }^{2}$ School of Physics and Astronomy, University of St Andrews, St Andrews KY16 9AJ, UK \\ ${ }^{3}$ Department of Astronomy, Columbia University, New York, NY 10027, USA \\ ${ }^{4}$ MIT-Kavli Center for Astrophysics and Space Research, 77 Massachusetts Avenue, Cambridge, MA 02139, USA \\ Received 2017 February 13; revised 2017 July 12; accepted 2017 July 17; published 2017 September 12
}

\begin{abstract}
We report on observations made with the Cosmic Origins Spectrograph (COS) on the Hubble Space Telescope (HST) using background quasi-stellar objects to probe the circum-galactic medium (CGM) around 17 low-redshift galaxies that are undergoing or have recently undergone a strong starburst (the COS-Burst program). The sightlines extend out to roughly the virial radius of the galaxy halo. We construct control samples of normal star-forming low-redshift galaxies from the COS/HST archive that match the starbursts in terms of galaxy stellar mass and impact parameter. We find clear evidence that the CGM around the starbursts differs systematically compared to the control galaxies. The $\mathrm{Ly} \alpha, \mathrm{Si}$ III, C IV, and possibly O VI absorption lines are stronger as a function of impact parameter, and the ratios of the equivalent widths of C IV/Ly $\alpha$ and Si III/Ly $\alpha$ are both higher than in normal starforming galaxies. We also find that the widths and the velocity offsets (relative to $v_{\text {sys }}$ ) of the Ly $\alpha$ absorption lines are significantly larger in the CGM of the starbursts, implying velocities of the absorbing material that are roughly twice the halo virial velocity. We show that these properties can be understood as a consequence of the interaction between a starburst-driven wind and the preexisting CGM. These results underscore the importance of winds driven from intensely star-forming galaxies in helping drive the evolution of galaxies and the intergalactic medium. They also offer a new probe of the properties of starburst-driven winds and of the CGM itself.
\end{abstract}

Key words: galaxies: halos - galaxies: ISM - galaxies: starburst - quasars: absorption lines

\section{Introduction}

The evolution of galaxies is largely driven by how and when they accrete gas and by how the feedback from newly formed stars and black holes regulates this accretion (see Somerville \& Davé 2015 and references therein). In turn, the evolution of the intergalactic medium will be affected by these same feedback processes, which can photoionize, shock-heat, and chemically enrich it (e.g., Ménard et al. 2010). These flows into and out of galaxies will occur within the circum-galactic medium (CGM), a region extending out to roughly the galaxy virial radius. Over the past several years, Hubble Space Telescope (HST)/ Cosmic Origins Spectrograph (COS) observations have greatly improved our understanding of the properties of the CGM in low- $z$ galaxies (e.g., Stocke et al. 2013; Tumlinson et al. 2013; Bordoloi et al. 2014b; Liang \& Chen 2014; Werk et al. 2014; Borthakur et al. 2015, 2016; Johnson et al. 2015). We now know that the CGM of both star-forming and quiescent galaxies contains a significant reservoir of gas clouds or filaments, most likely at $T \sim 10^{4} \mathrm{~K}$ and photoionized by the diffuse meta-galactic UV background. The CGM in normal star-forming galaxies also contains highly ionized gas traced by $\mathrm{O}$ VI that is only rarely present in the CGM of quiescent galaxies (Tumlinson et al. 2011).

One of the main ways in which feedback occurs is via the outflows of gas driven from strongly star-forming galaxies by the energy and/or momentum injected by massive stars (see Heckman \& Thompson 2017 for a recent review). These galactic winds are ubiquitous in star-forming galaxies at intermediate and high redshift (e.g., Weiner et al. 2009; Steidel

\footnotetext{
Hubble Fellow.
}

et al. 2010; Erb et al. 2012; Kornei et al. 2012; Martin et al. 2012; Bordoloi et al. 2014a; Rubin et al. 2014), while in the present-day universe, winds are only observed from starburst galaxies-objects with high star formation rates (SFRs) per unit area and/or per unit mass (e.g., Chisholm et al. 2015; Heckman et al. 2015; Heckman \& Borthakur 2016; Ho et al. 2016). In principle, galactic winds can have dramatic effects. They may account for the high relative mass of metals in the IGM, for the evolving mass-metallicity relation for galaxies, for the expulsion of baryons from low-mass dark matter halos, and for the transport of material with low angular momentum from forming galaxies (Somerville \& Davé 2015 and references therein).

Unfortunately, these effects have yet to be robustly quantified through direct observation. The principal problems are that (1) the winds are complex multiphase flows whose physical properties and effects on their surroundings can be fully probed through the detailed multiwaveband observations that are possible only in local galaxies. (2) Observations of such outflows from local star-forming galaxies have generally been limited to regions either inside the main body of the galaxy or in the innermost parts of the CGM (on a scale of $10 \mathrm{kpc}$ or less). Given that the directly measured outflow speeds in these regions are typically comparable to the galaxy escape velocity, it is not clear whether or how these outflows affect the bulk of the CGM.

To date, there has been only a small amount of data testing whether galactic winds from starburst galaxies at low redshift could have a major impact on the CGM. Borthakur et al. (2013; hereafter B13) used data taken in a pilot program of observations of background quasi-stellar objects (QSOs) with COS to show that the CGM around a small sample of five 
low- $z$ starburst and post-starburst (SB/PSB) galaxies often have strong $\mathrm{C}$ IV absorption lines arising in a CGM that extends out to impact parameters of $\sim 200 \mathrm{kpc}$. Lines this strong are not seen in the outer CGM of low-redshift normal star-forming or quiescent galaxies (e.g., Liang \& Chen 2014). The implied masses and densities of this highly ionized material are similar to what has been inferred for the much cooler photoionized clouds/filaments seen in the halos of more typical low- $z$ galaxies. Thus, we argued that these results can be understood as the consequence of a starburst-driven wind that has propagated far out into the CGM, interacting with the preexisting cooler clouds/filaments.

In the present paper, we report on the COS-Burst program: new observations that represent a major improvement on the B13 results. We have significantly expanded the size of the sample of low- $z$ starburst/post-starbursts (from 5 galaxies to 17), allowing us to investigate the wind-CGM interaction in a much more statistically robust way. Second, we can now characterize the properties of the CGM in SB/PSB galaxies using multiple ions (not just C IV). In particular, in six cases we can measure the $\mathrm{O}$ VI absorption lines, extending our probe of the CGM to hotter gas than before. Our larger COS-Burst sample also allows us to explore the radial dependence of the CGM properties on the properties of the SB/PSB galaxies.

\section{Observations}

\subsection{Sample Selection}

We have developed a technique based on principal component analysis (PCA) for identifying starbursts and post-starburst galaxies using SDSS DR7 spectra (Wild et al. 2007, 2010). The amplitude of the first principal component (PC1) essentially measures the strength of the $4000 \AA$ break (a probe of the specific SFR over the past few Gyr). The amplitude of the second principal component (PC2) measures the strength/weakness of the high-order Balmer absorption lines relative to the strengths and weaknesses in an average galaxy with the same value of PC1. Combining these two gives us a diagnostic diagram for selecting and characterizing galaxies that are undergoing or have recently undergone a strong burst of star formation (typically involving at least $10 \%$ of the galaxy stellar mass). This is shown in Figure 1, where we also show the time-dependent trajectory of models of such starbursts and their descendants (post-starbursts) over a period of about $600 \mathrm{Myr}$. A montage of SDSS images of the COSBurst sample is shown in Figure 2, showing them to mostly be normal late-type galaxies in terms of morphology.

After selecting a sample of SDSS galaxies lying along this trajectory, we then cross-matched this sample with the SDSS sample of QSOs in the GALEX GR6 catalog. Our final sample consists of those SB/PSB galaxies having a background QSO with FUV $<19$ lying along a sightline passing less than $230 \mathrm{kpc}^{6}$ from the foreground galaxy. The properties of the individual galaxies in this sample are listed in Table 1, while the median values for the sample as a whole are given in Table 2.

The geometry of the sightlines probed by the selected COSBurst targets is plotted in Figure 3. For this figure we use a normalized impact parameter $\left(\rho / R_{\mathrm{vir}}\right)$, where $R_{\mathrm{vir}}$ is the halo virial radius. We describe below how we determine $R_{\text {vir. }}$ By

6 We adopt $H_{0}=70 \mathrm{~km} \mathrm{~s}^{-1} \mathrm{Mpc}^{-1}, \Omega_{\Lambda}=0.7$, and $\Omega_{M}=0.3$.

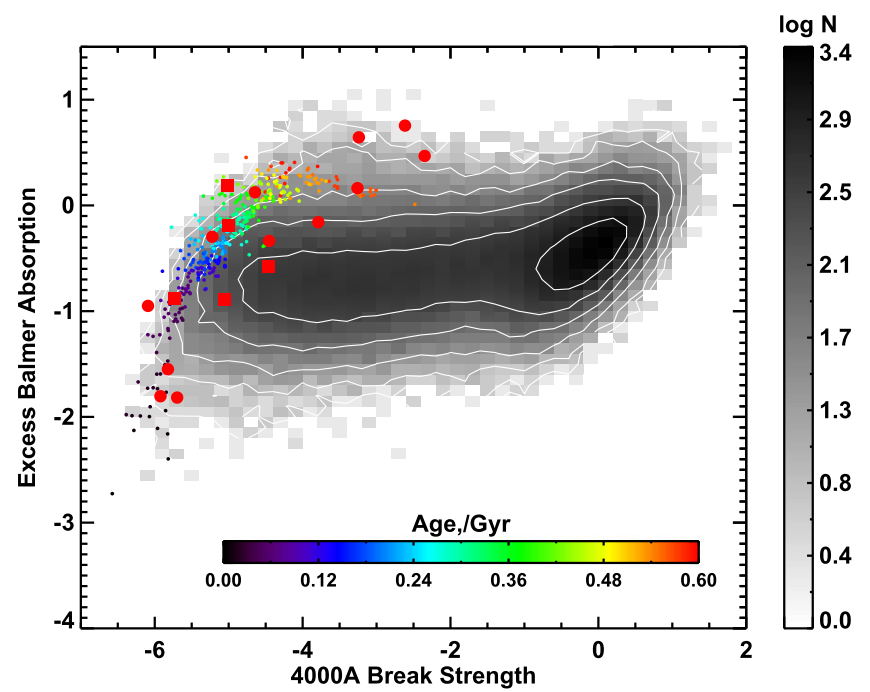

Figure 1. Plot used to identify the COS-Burst sample from SDSS spectra. The plotted parameters are based on a PCA-based analysis, and represent the strength of the $4000 \AA$ break (PC1, on the $x$-axis) and the excess strength of the high-order Balmer absorption lines (PC2, on the $y$-axis), both in dimensionless units. The gray scale indicates the relative numbers of all SDSS galaxies with the luminosity-weighted mean age of their stellar population increasing from left to right. The small color-coded dots show a model library of starbursts and post-starbursts with typical burst-mass fractions of $10 \%-20 \%$, durations (e-folding times) of a few hundred million years, and ages increasing from bottom to top. Our COS-Burst sample members are shown as large red symbols.

chance, we have no sightlines located within $\sim 30^{\circ}$ of the galaxy major axis and so cannot probe the effect of the starburst on CGM material near the disk plane. We also primarily sample the outer CGM $\left(\rho>0.5 R_{\text {vir }}\right)$.

\subsection{Generation of Control Samples}

In our analysis we emphasize differential measurements in which each property of CGM of the COS-Burst galaxies is compared to a control sample of normal galaxies. As shown by Borthakur et al. (2016; hereafter B16), there are systematic differences in CGM properties between low-redshift starforming (blue) and quiescent (red) galaxies (and see Ménard et al. 2011 and Bordoloi et al. 2011 for galaxies at intermediate redshifts). Since the COS-Burst galaxies would most likely have been star-forming galaxies before the starburst, we assess the effect of the starburst using only star-forming galaxies in the control samples. ${ }^{7}$ For the same reason, the control samples were selected to cover the same range in stellar mass $\left(M_{*}\right)$ as the COS-Burst sample $\left(\sim 10^{10}-10^{11} M_{\odot}\right.$, see Tables 1 and 2$)$. Finally, there are systematic radial declines in the equivalent widths of the CGM absorption lines (e.g., Prochaska et al. 2011; Bordoloi et al. 2014b; Liang \& Chen 2014; Johnson et al. 2015; Borthakur et al. 2015; B16). We therefore selected control samples with distributions of $\rho / R_{\mathrm{vir}}$ that are similar those listed in Table 1 and plotted in Figure 3.

In the analysis below we focus on the properties of the following transitions (which represent the strongest and most commonly detected lines in our data): Ly $\alpha$, Si III 1206.5, C IV 1548.2, and O VI 1031.9. Unfortunately, there is no single control sample that can be constructed from the $H S T / \mathrm{COS}$ archive that can be used for all four transitions. For Ly $\alpha$ and

\footnotetext{
7 If we had used the quiescent red galaxies instead, the differences we find below would be even larger (B16).
} 

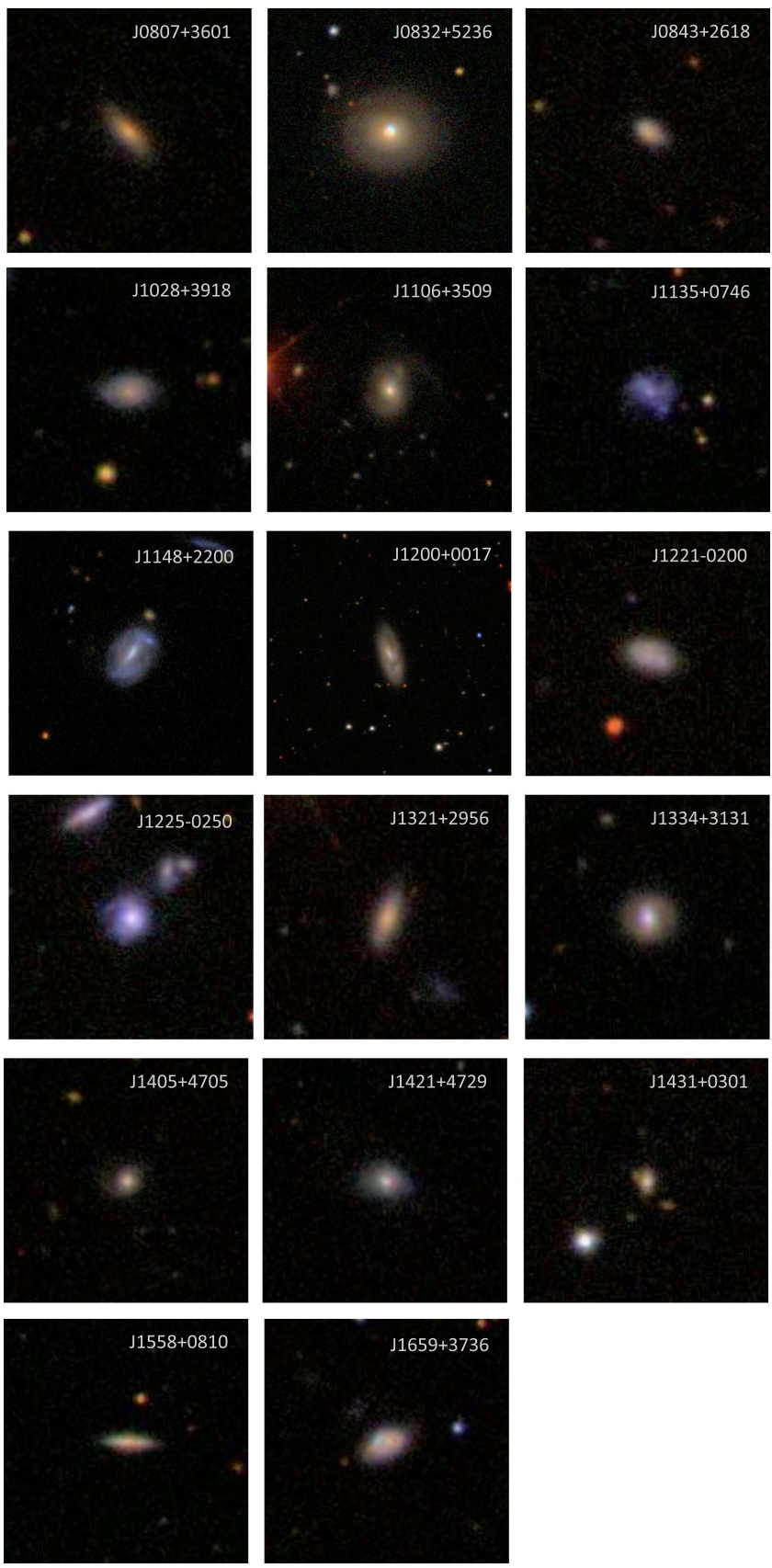

Figure 2. Multicolor SDSS composite image of our sample of 17 COS-Burst galaxies. The galaxies are identified by their name in the top right corner. Details of our sample are presented in Table 1.

Si III we use the sample of star-forming galaxies analyzed by B16 (drawn from the COS-Halos and COS-GASS programs). For CIV we combine the data for the normal star-forming galaxies in B13 with those in the sample presented in the compilation in Liang \& Chen (2014) that lie in the same range of stellar mass and normalized impact parameter as the COSBurst sample. For O VI, we select the star-forming galaxies from the compilation of Johnson et al. (2015) spanning the same ranges in stellar mass and normalized impact parameter as the COS-Burst sample.

The properties of these control samples are compared to those of the COS-Burst sample in Table 2. We describe how these properties were measured for COS-Burst and the control samples in Section 2.4 below. Table 2 shows that the control samples are good matches to the COS-Burst sample in almost all respects (median values of $M_{*}, v_{c}, R_{\mathrm{vir}}, \rho$, and galaxy halflight radius $\left.\left(R_{50}\right)\right)$. The only exception is the O VI control sample, where the median impact parameter is only $56 \%$ as large as for the COS-Burst sample.

As noted above, none of the COS-Burst sightlines lie within $30^{\circ}$ of the galaxy major axis. This might bias the comparison to the control samples. For a number of reasons, this should not be a significant effect. First, for the 11 cases in the COS-Burst sample where we can measure the orientation of the COS sightline, we would only have expected 3.7 targets with $\Theta<30^{\circ}$. This only represents $22 \%$ of the sample of 17 . Second, over the range of azimuthal angles we do probe $\left(\Theta=30^{\circ}-90^{\circ}\right)$, we see no variation in CGM properties. Third, for the control sample used to compare the properties of the Ly $\alpha$ and Si III lines, Borthakur et al. (2015) showed that there is no azimuthal dependence for the structure of the outer CGM (the region probed in the COS-Burst sample). Finally, while an azimuthal dependence of the strength of the $\mathrm{Mg}$ II absorption line has been seen in the CGM of star-forming galaxies (Bordoloi et al. 2011; Bouché et al. 2012; Kacprzak et al. 2012; Ho et al. 2016), these sightlines are typically much closer to the disk of the galaxy than in our samples (mean impact parameters of $<50 \mathrm{kpc}, 36 \mathrm{kpc}, 48 \mathrm{kpc}$, and $53 \mathrm{kpc}$ for these four studies, respectively).

\subsection{Analysis of COS Data}

The new COS-Burst data (Program 13862) were obtained using the COS FUV G130M and G160M gratings, yielding spectral resolutions of 15,000 and 18,000 , respectively (20 and $18 \mathrm{~km} \mathrm{~s}^{-1}$ FWHM). The program was designed so that (when combined with the data in B13) we covered the Si III 1206.5 and Ly $\alpha$ lines in all 17 cases, the C IV 1548.2,1550.8 doublet in 16 cases, and the O VI 1031.9, 1037.6 doublet for the 6 cases with redshifts $z>0.073$ (placing O VI 1031.9 longward of $\sim 1107 \AA$ ).

The data were reduced and analyzed following the procedure described in B13, and we refer the reader there for details. We characterized the absorption line profiles using three nonparametric properties: the equivalent width, the absorbed-fluxweighted mean wavelength (centroid), and the FWHM (of the absorbed) intensity. The equivalent widths were then converted into the rest-frame values, the flux-weighted line centroid was used to calculate the velocity difference between the line and the galaxy systemic velocity (based on SDSS spectra), and the line width was converted into $\mathrm{km} \mathrm{s}^{-1}$. We henceforth refer to these three quantities as equivalent widths (EW), $\Delta v$, and FWHM, respectively. We have also reanalyzed the COS G140M data for the SB/PSB galaxies in B13 to measure these same parameters. The results are listed in Table 3.

\subsection{Parameters from Ancillary Data}

In this paper we use a number of parameters to characterize the COS-Burst and control galaxies and the QSO sightlines through the CGM (as listed in Tables 1 and 2). The galaxy stellar masses $\left(M_{*}\right)$ for the COS-Burst and the Ly $\alpha / \mathrm{Si}$ III control samples were taken from the MPA-JHU value-added catalog, calculated using the method published by Salim et al. (2007). The stellar masses for the galaxies in the C IV and O VI samples were taken from B13, Liang \& Chen (2014), and Johnson et al. (2015). The B13 masses were determined in the 
Table 1

Description of Galaxy Properties

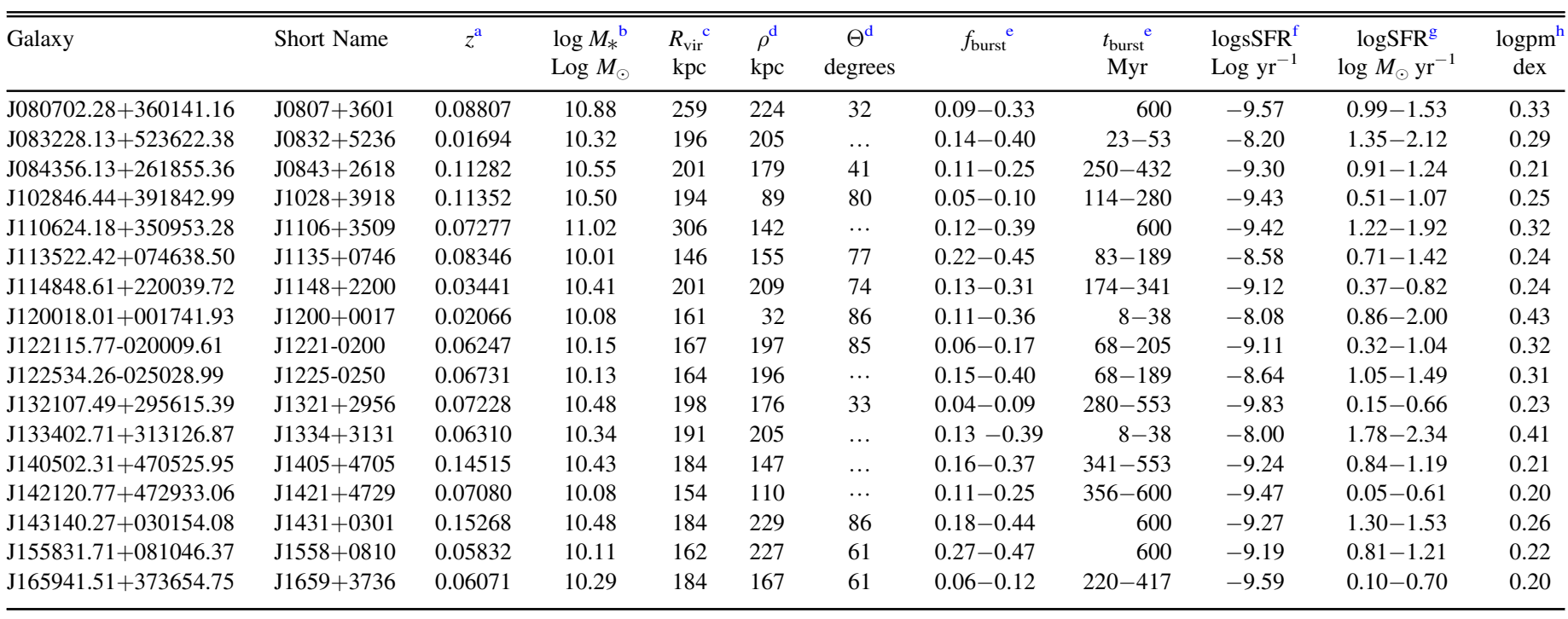

Notes.

a The galaxy redshift from SDSS.

b The total galaxy stellar mass from the MPA-JHU Value-Added Galaxy Catalog.

${ }^{c}$ The galaxy halo virial radius, based on the stellar mass. See the text for details.

${ }^{d}$ The impact parameter $(\rho)$ and orientation with respect to the galaxy major axis $(\Theta)$ for the sightline through the CGM. No value is given for $\Theta$ in face-on cases.

${ }^{\mathrm{e}}$ The burst-mass fraction and burst age based on PCA analyses of the SDSS spectra. See the text for details. The quoted range represents the 16 to 84 percentile values for the probability distribution functions.

${ }^{\mathrm{f}}$ The $\log$ of the effective specific SFR for the burst, defined as $f_{\text {burst }} / t_{\text {burst }}$.

g The log of the SFR. The quoted range represents no aperture correction to the SDSS fiber (minimum value) and SFR $=$ sSFR $M_{*}$ (maximum value).

${ }^{\mathrm{h}}$ The uncertainties in $\log$ SSFR and $\log$ SFR due to the uncertainties in $f_{\text {burst }}$ and $t_{\text {burst }}$.

same way as for the COS-Burst sample, while the other masses were based on the NASA-Sloan Galaxy Atlas (http://nsatlas. org/), using a similar method.

The stellar mass was used to estimate the mass of the dark matter halo, following the method described in B16 for starforming galaxies (which was based on the analyses of Kravtsov et al. 2014; Liang \& Chen 2014; Johnson et al. 2015, and Mandelbaum et al. 2016). Following Liang \& Chen (2014) and Johnson et al. (2015), we have used the halo mass to determine the virial radius $\left(R_{\mathrm{vir}}\right)$ using Equation (3) in Liang \& Chen (2014). This ensures that we have determined the virial radius for the COS-Burst sample in the same way as the control samples

We have then defined a normalized impact parameter for each sightline $\left(\rho_{n}\right)$, defined as the ratio of the impact parameter $\rho$ and $R_{\text {vir }}$. We also specified the orientation of the sightline with respect to the galaxy major axis (as measured based on SDSS images). Here $\Theta=0(90)^{\circ}$ corresponds to a sightline along the galaxy major (minor) axis.

To determine the parameters of the starburst in the COSBurst sample, we use the SDSS spectra and employ the method described in Wild et al. (2010). The starbursts are modeled as events with exponentially declining SFRs. Good fits that also include fitting the observed $\mathrm{H} \alpha$ emission-lines required timeconstants $(\tau)$ of $\sim 200-350$ Myr. The Bayesian analysis yields probability distribution functions for the fraction of the galaxy stellar mass involved in the burst $\left(f_{\text {burst }}\right)$ and the time since the burst began ( $\left.t_{\text {burst }}\right)$. We use the median values of the distributions and characterize the uncertainties using the 16 and 84 percentiles in the distribution. While the oldest bursts can be identified, the values of the burst age are not well constrained for $t_{\text {burst }}>600$ Myr. We define a characteristic SFR averaged over the burst as $S F R=f_{\text {burst }} M_{*} / t_{\text {burst }}$ and a characteristic specific SFR (sSFR) per unit mass as $s S F R=f_{\text {burst }} / t_{\text {burst }}$ in units of inverse years. The uncertainties on these derived parameters are typically $\sim \pm 0.3$ dex (see Table 1 ), based on the probability distribution functions for $f_{\text {burst }}$ and $t_{\text {burst }}$. We only use these values to determine the typical (median) values for the sample as a whole (as listed in Table 2).

\section{Results}

\subsection{Overview}

Of the transitions lying in our spectral coverage, we detect the O VI 1031.9, 1037.6, and C IV 1548.2, 1550.8 doublets, and the Si III 1206.5 and Ly $\alpha$ lines. We have only upper limits for Si II 1260.4, Ci II 1334.5, and Si IV 1393.8. For these nondetections we have used signal-to-noise-weighted stacked spectra to set upper limits (Table 4).

For the four detected features, the detection fraction varies. The Ly $\alpha$ line is detected in 16 of the 17 SB/PBS sightlines with contamination by the $\mathrm{OI}$ telluric airglow line in $\mathrm{J} 1421+47$. This implies an effective detection fraction of $f_{\text {det }}=100 \%$. The Si III line is detected in 8 of the 17 sightlines $\left(f_{\mathrm{det}}=47 \%\right)$. The C IV line is detected in 7 of 16 sightlines $\left(f_{\text {det }}=44 \%\right)$. We only probe O VI along 6 sightlines, with 3 detections $\left(f_{\text {det }}=50 \%\right)$. We note that detections of the different metal lines are strongly related to one another. Five of the $7 \mathrm{Si}$ III detections are detected in C IV, while none of the 9 Si III non-detections are. Similarly, 2 of the 3 O VI detections have detections in $\mathrm{CIV}$, while none of the 3 non-detections do. 
Table 2

Median Properties of the Samples

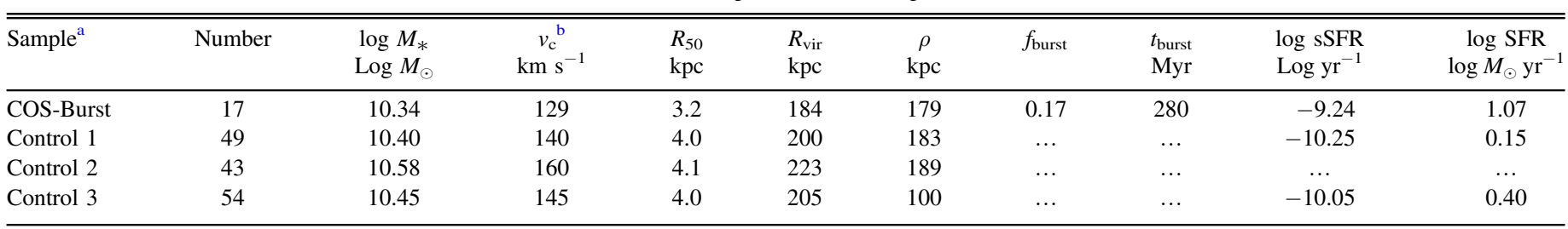

Notes.

${ }^{\text {a }}$ Control sample 1 was used to compare the properties of the Ly $\alpha$ and Si III lines. Control samples 2 and 3 were for C IV and O VI, respectively. See the text.

${ }^{b}$ The median value of the characteristic circular velocity of the halo based on the halo mass and virial radius.

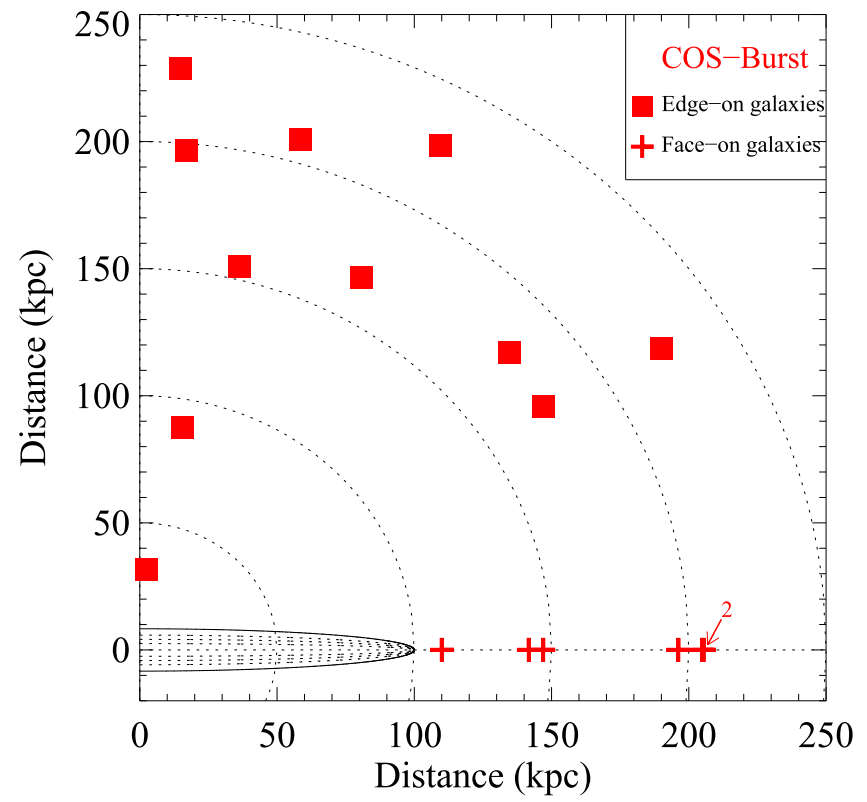

Figure 3. Distribution of the COS-Burst sightlines through the CGM. The orientation of the major axis of the galaxy is shown at the origin (not to scale). In six cases the galaxy is seen close to face-on, and these sightlines are plotted as crosses with $y=0$. We have good coverage of the outer CGM. We do not have sightlines near the galaxy disk plane for the 11 inclined galaxies.

The Ly $\alpha$ lines are optically thick (saturated), so we do not measure H I column densities. For the detected Si III 1206.5, C IV 1548.2, and O VI 1031.9 lines the median (mean) optical depths in the line cores derived from fitting Voigt profiles are $\sim 0.9$ (1.1). These optical depths are consistent with the median (mean) value of the CIV 1548.2/1550.8 and O IV 1031.9/ 1037.6 doublet ratios of 1.73 (1.68), which imply $\tau \sim 1$ for the stronger member of the doublet in both cases.

Considering only the detected systems, the mean column densities are $N_{\mathrm{O} \text { VI }}=10^{14.8}, \quad N_{\mathrm{C} \text { IV }}=10^{14.7}$, and $N_{\mathrm{Si} \text { III }}=$ $10^{13.5} \mathrm{~cm}^{-2}$. For species that were not detected, the upper limits based on the stacked spectra (see Table 4) imply column densities of Si II, Ci II, and Si IV are $<10^{12.9},<10^{13.4}$, and $<10^{13.1} \mathrm{~cm}^{-2}$, respectively.

\subsection{Starbursts versus Controls}

\subsubsection{Radial Distributions}

In Figure 4 we plot the radial distributions of the rest-frame EW of the Ly $\alpha$, Si III 1206.5, C IV 1548.2, and O VI 1031.9 lines as a function of normalized impact parameter $\left(\rho / R_{\text {vir }}\right)$. In each case, we compare the COS-Burst sample to the relevant control sample described above. We overplot the best fit to the radial dependence of the equivalent width as determined for both the COS-Burst and star-forming (control) galaxies. All these fits make explicit use of the upper limits (see B16 for details). Following B16, we define the excess Ly $\alpha$ equivalent widths as the difference between the logarithms of the measured equivalent width and of the equivalent width at the corresponding normalized impact parameter based on the fit to the control sample.

It is immediately clear that the COS-Burst sample is systematically displaced toward stronger absorption lines than the control samples in the cases of Ly $\alpha, \mathrm{Si}$ III, and C IV. The results for O VI are consistent with this, but the small sample size limits the statistical significance. These results pertain mainly to the outer CGM as a result of the paucity of COSBurst sightlines in the inner CGM.

\subsubsection{Kinematics}

Since it has the largest number of detections, we have used the $\operatorname{Ly} \alpha$ line to characterize the kinematics of the CGM. We did this using two quantities defined above: (1) $\Delta v=\left|v_{\mathrm{Ly} \alpha}-v_{\text {sys }}\right|$ (where $v_{\text {sys }}$ is the systemic velocity of the galaxy based on SDSS), and (2) the FWHM defined as the nonparametric FWHM of the absorption line profile. We have measured these parameters for the COS-Burst sample and for the control sample of star-forming galaxies in COS-GASS (see Borthakur et al. 2015).

In Figure 5 we plot histograms of $\Delta v$ and FWHM for the COS-Burst and control samples. The COS-Burst sample is offset to higher values than the control sample (by median values of $\sim 0.3$ dex in FWHM and $\sim 0.2$ dex in $\Delta v$ ). These differences are significant at $>99.99 \%$ confidence levels according to a Wilcoxon rank test.

To gain more insight into the kinematic properties of the CGM in the COS-Burst sample, we have measured the FWHM of the Ly $\alpha$, Si III, C IV, and O VI absorption lines using the signal-to-noise-weighted stacked spectra for each transition. These stacks were created by aligning the individual spectra using the systemic velocity of the galaxy from SDSS. These stacked spectra then show the velocity range covered by the absorbing gas in the entire sample (including both the bulk offsets in velocity and the line-of-sight velocity spreads seen in the individual spectra). The resulting Ly $\alpha$ profile is shown in Figure 6. The FWHM of this line is $424 \pm 20 \mathrm{~km} \mathrm{~s}^{-1}$, and this is consistent with the average value for the noisier Si III, $\mathrm{C}$ IV, and OVI stacked profiles $\left(366 \pm 45 \mathrm{~km} \mathrm{~s}^{-1}\right)$. The corresponding stacked Ly $\alpha$ profile for the COS-GASS plus COS-Halos control sample (Table 2) is much narrower $\left(210 \pm 30 \mathrm{~km} \mathrm{~s}^{-1}\right)$. 
Table 3

Absorption Line Properties ${ }^{\mathrm{a}}$

\begin{tabular}{|c|c|c|c|c|c|c|}
\hline Short Name & $\begin{array}{c}\mathrm{EQ}_{\mathrm{Ly} \alpha} \\
\mathrm{mA}\end{array}$ & $\begin{array}{c}\mathrm{EQ}_{\mathrm{Si} \text { III }} \\
\mathrm{m \AA}\end{array}$ & $\begin{array}{c}\mathrm{EQ}_{\mathrm{C}} \mathrm{IV} \\
\mathrm{m \AA}\end{array}$ & $\begin{array}{c}\mathrm{EQ}_{\mathrm{O}} \mathrm{vI} \\
\mathrm{m \AA}\end{array}$ & $\begin{array}{c}\mathrm{FWHM}^{\mathrm{b}} \\
\mathrm{km} \mathrm{s}^{-1}\end{array}$ & $\begin{array}{c}\Delta v^{\mathrm{c}} \\
\mathrm{km} \mathrm{s}^{-1}\end{array}$ \\
\hline $\mathrm{J} 0807+3601$ & $925 \pm 66$ & $<132$ & $689 \pm 127$ & $542 \pm 124$ & $231 \pm 16$ & $160 \pm 75$ \\
\hline $\mathrm{J} 0832+5236$ & $836 \pm 36$ & $354 \pm 89$ & $398 \pm 121$ & $\ldots$ & $212 \pm 31$ & $54 \pm 16$ \\
\hline $\mathrm{J} 0843+2618$ & $507 \pm 60$ & $<144$ & $<160$ & $175 \pm 68$ & $116 \pm 12$ & $93 \pm 29$ \\
\hline $\mathrm{J} 1028+3918$ & $1280 \pm 67$ & $231 \pm 48$ & $1220 \pm 72$ & $912 \pm 122$ & $312 \pm 31$ & $15 \pm 24$ \\
\hline $\mathrm{J} 1106+3509$ & $437 \pm 23$ & $<63$ & $<273$ & $<246$ & $100 \pm 20$ & $17 \pm 56$ \\
\hline $\mathrm{J} 1135+0746$ & $718 \pm 61$ & $<96$ & $<519$ & $<402$ & $185 \pm 21$ & $77 \pm 25$ \\
\hline $\mathrm{J} 1148+2200$ & $664 \pm 123$ & $340 \pm 120$ & $<264$ & $\ldots$ & $320 \pm 20$ & $172 \pm 22$ \\
\hline $\mathrm{J} 1200+0017$ & $1148 \pm 152$ & $843 \pm 275$ & $1099 \pm 219$ & $\ldots$ & $289 \pm 45$ & $63 \pm 34$ \\
\hline J1221-0200 & $497 \pm 88$ & $<250$ & $828 \pm 55$ & $\ldots$ & $143 \pm 73$ & $81 \pm 40$ \\
\hline $\mathrm{J} 1225-0250$ & $898 \pm 44$ & $650 \pm 50$ & $560 \pm 75$ & $\ldots$ & $199 \pm 20$ & $74 \pm 19$ \\
\hline $\mathrm{J} 1321+2956$ & $275 \pm 75$ & $<200$ & $<495$ & $\ldots$ & $399 \pm 150$ & $97 \pm 78$ \\
\hline $\mathrm{J} 1334+3131$ & $1095 \pm 23$ & $<423$ & $<546$ & $\ldots$ & $285 \pm 10$ & $412 \pm 64$ \\
\hline $\mathrm{J} 1405+4705$ & $1950 \pm 50$ & $220 \pm 60$ & $582 \pm 69$ & $\ldots$ & $412 \pm 30$ & $25 \pm 85$ \\
\hline $\mathrm{J} 1421+4729$ & $\ldots$ & $<171$ & $<618$ & $\ldots$ & $\ldots$ & $\ldots$ \\
\hline $\mathrm{J} 1431+0301$ & $938 \pm 226$ & $230 \pm 100$ & $\ldots$ & $<184$ & $431 \pm 40$ & $39 \pm 42$ \\
\hline $\mathrm{J} 1558+0810$ & $605 \pm 109$ & $<243$ & $<459$ & $\ldots$ & $154 \pm 10$ & $255 \pm 27$ \\
\hline $\mathrm{J} 1659+3736$ & $1032 \pm 269$ & $288 \pm 154$ & $<357$ & $\ldots$ & $270 \pm 25$ & $99 \pm 18$ \\
\hline
\end{tabular}

Notes.

a These are the rest-frame equivalent widths for the Ly $\alpha$, Si III 1206.5, C IV 1548.2, and O VI 1031.9 lines.

b The nonparametric FWHM of the Ly $\alpha$ absorption feature.

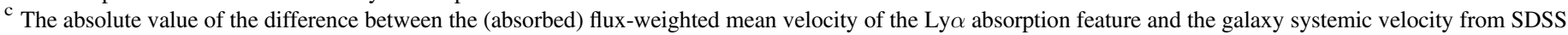
spectra. The quoted uncertainties include those in both the Ly $\alpha$ centroid and the SDSS galaxy systemic velocity.

Table 4

Rest Equivalent Widths from Stacked Spectra ${ }^{a}$

\begin{tabular}{lcccccc}
\hline \hline Ly $\alpha$ & Si III & C IV & O VI & Si II & C II & Si IV \\
\hline $756 \pm 62$ & $165 \pm 43$ & $326 \pm 80$ & $300 \pm 100$ & $<137$ & $<56$ & $<109$ \\
\hline
\end{tabular}

Note.

' The specific transitions are Ly $\alpha$, Si III 1206.5, C IV 1548.2, O VI 1031.9, Si II 1260.4, Ci II 1334.5, and Si IV 1393.8. The values are all in $m \AA$.

To place these velocities into context, we can compare the line widths to expectations for profiles produced by a population of clouds moving randomly through the CGM at the circular virial velocity $\left(v_{c}\right)$ of the dark matter halo. For the COS-Burst sample the median value of $v_{c}$ is $129 \mathrm{~km} \mathrm{~s}^{-1}$ (Table 2). In this case, and assuming the halo potential is an isothermal sphere (Binney \& Tremaine 1987), the implied FWHM of the line profile would be $214 \mathrm{~km} \mathrm{~s}^{-1}$. This is only about half as wide as the observed Ly $\alpha$ profile, implying that the observed velocities are super-virial. In contrast, for the control sample, the median value for $v_{c}$ $\left(140 \mathrm{~km} \mathrm{~s}^{-1}\right)$ would imply an FWHM $=233 \mathrm{~km} \mathrm{~s}^{-1}$, consistent with the observed profile.

\subsubsection{Line Ratios}

From Figure 4, it is clear that the differences between the CGM in the COS-Burst galaxies and the normal galaxies are stronger in the Si III and C IV equivalent widths than in Ly $\alpha$. This is shown more explicitly in Figure 7, where we compare histograms of the ratios of the $\mathrm{Si}$ III/Ly $\alpha$ and $\mathrm{CIV} / \mathrm{Ly} \alpha$ equivalent widths between the two samples.

These differences are consistent with our stacking results and those of Liang \& Chen (2014) for normal star-forming galaxies. For our stacked COS-Burst spectra (Table 4) we find $\log \left(\mathrm{EQ}_{\mathrm{C} \text { IV }} / \mathrm{EQ}_{\mathrm{Ly} \alpha}\right)=-0.37 \pm 0.06$ and $\log \left(\mathrm{EQ}_{\mathrm{Si} \text { III }} /\right.$ $\left.\mathrm{EQ}_{\mathrm{Ly} \alpha}\right)=-0.66 \pm 0.11$. The corresponding values from Liang
$\&$ Chen (for the radial bin $\rho=0.56$ to $1.09 R_{\mathrm{vir}}$ ) are $-0.78 \pm 0.20$ and $-1.08 \pm 0.23$ (lower than the COS-Burst values by $\sim 0.4$ dex).

The physical meaning of the higher ratios of the $\mathrm{Si}$ III $/ \mathrm{L} \alpha$ and $\mathrm{CIV} / \mathrm{Ly} \alpha$ equivalent widths in the CGM of the COSBurst galaxies is not straightforward. The Ly $\alpha$ absorption lines in all the COS-Burst sightlines and the majority of the control sample sightlines are saturated (highly optically thick). In these cases, the $\operatorname{Ly} \alpha$ equivalent width is primarily tracing the spread in velocity of the absorbing gas along the line of sight (rather than column density). In contrast, the Si III and C IV lines have typical optical depths of about 1 (see above). Their equivalent widths therefore trace the ionic column densities in the CGM. The enhanced ratios in the COS-Burst sample presumably reflect higher overall gas column densities through the CGM (which increase the strength of the unsaturated lines (C IV and Si III) relative to the saturated lines $(\operatorname{Ly} \alpha)$.

\subsection{The Metal Content of the CGM}

In the following we estimate the mass of various metal ions using the absorption line data and the measured column densities. For simplicity, we take the average column density for a given species based on the detections and then multiply this by the fraction of sightlines along which detections were made. We then multiply this effective column density by the geometrical cross-sectional area of the outer CGM to obtain an implied mass. The median virial radius in our sample was $184 \mathrm{kpc}$, and we calculate our masses using an annulus with inner and outer radii of 50 and $200 \mathrm{kpc}$.

We begin by considering silicon since we span a wide range of ionization states. The effective column densities are $<8 \times 10^{12}, 1.5 \times 10^{13}$, and $<1.2 \times 10^{13} \mathrm{~cm}^{-2}$ for Si II, Si III, and $\mathrm{Si}$ IV, respectively, The total implied silicon mass is $M_{\mathrm{Si}}=4.0$ to $10.0 \times 10^{5} M_{\odot}$ (assuming negligible $\mathrm{Si} \mathrm{V}$ or 

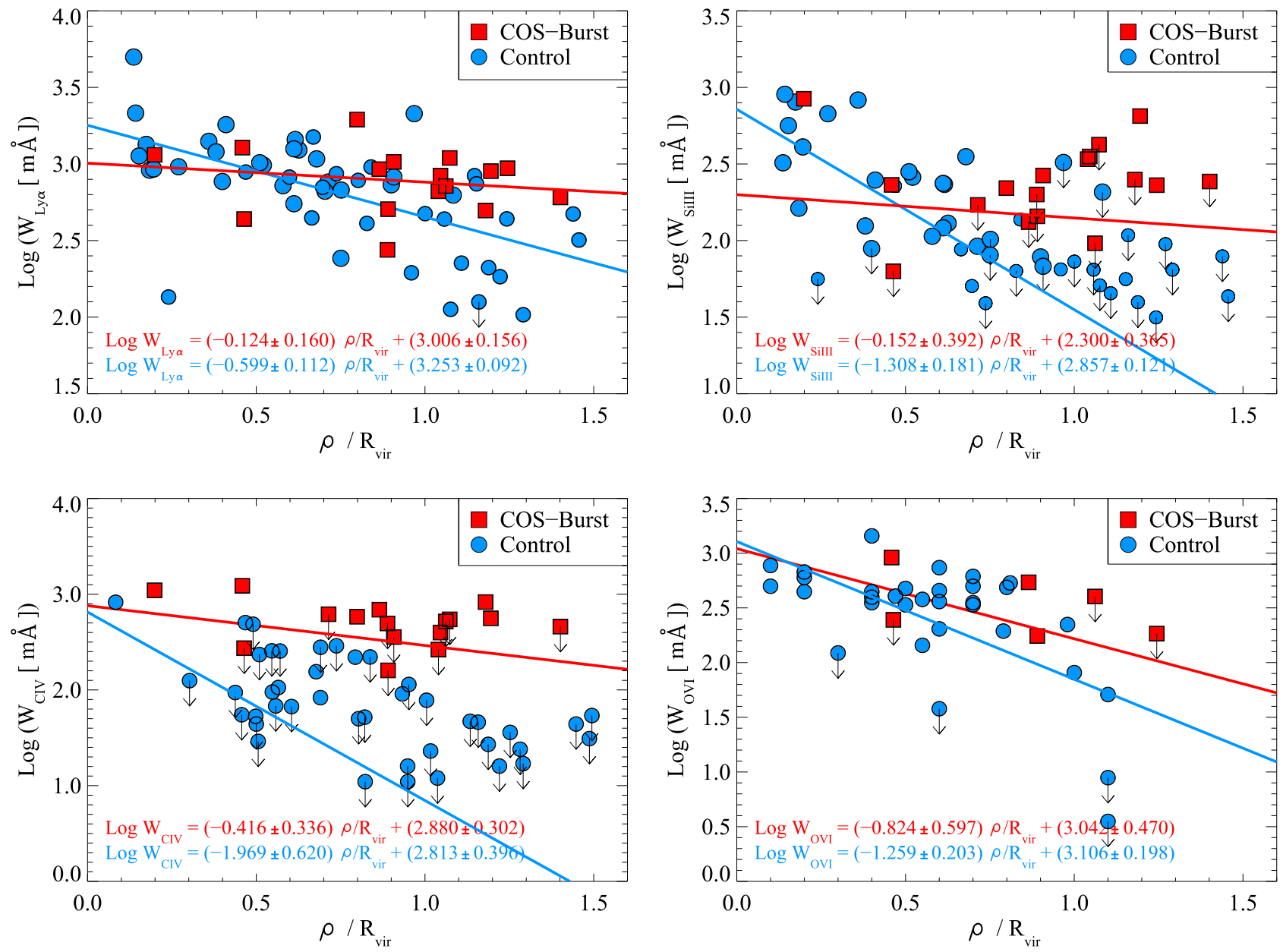

Figure 4. Log of the rest-frame equivalent widths of the Ly $\alpha$, Si III 1206.5, C IV 1548.2, and O VI 1031.9 absorption lines as a function of the normalized impact parameter $\left(\rho / R_{\mathrm{vir}}\right)$ of the sightline through the CGM. The COS-Burst sample of starburst/post-starburst galaxies are plotted as red squares, and the control samples of normal star-forming galaxies (see the text for details) are plotted as blue circles. In each panel we indicate the best-fit linear relations for both samples (calculated using both detections and upper limits). In all cases, the absorption lines are stronger in the outer CGM of the COS-Burst galaxies.

higher ions). For carbon we have effective column densities of $<2.5 \times 10^{13}$ and $2.2 \times 10^{14} \mathrm{~cm}^{-2}$ for $\mathrm{C}$ II and $\mathrm{C}$ IV, respectively, The implied mass is $M_{\mathrm{C} \text { IV }}=2.4 \times 10^{6} M_{\odot}$. Finally, we have an effective column density of $3.2 \times 10^{14} \mathrm{~cm}^{-2}$ for $\mathrm{O} \mathrm{VI}$, with an implied mass $M_{\mathrm{O} \text { vI }}=4.9 \times 10^{6} M_{\odot}$.

These masses can be compared to the metal mass of the warm CGM in normal star-forming galaxies determined by Peeples et al. (2014) for the COS-Halos sample (and see Bordoloi et al. 2014b). Peeples et al. find that the warm phase of the CGM (e.g., as traced by Si II, III, and Si IV) contains a total mass in all metals of $\sim 1.8 \times 10^{-3} M_{*}$. For the median value $M_{*}=2.2 \times 10^{10} M_{\odot}$ for our sample, the implied metal mass in the warm CGM would be $3.9 \times 10^{7} M_{\odot}$. Adopting a solar value for the Si-to-metal mass of 0.05 yields $M_{\mathrm{Si}}=1.9 \times 10^{6} M_{\odot}$, somewhat higher than we estimate in the outer CGM in our sample. The difference (as seen in Figure 4) is in the radial distributions: the metals assayed by Peeples et al. in normal star-forming galaxies are largely confined to the inner CGM (interior to $\sim 0.5 R_{\mathrm{vir}}$ ), whereas the metals detected in the CGM of the COS-Burst sample extend out to beyond the virial radius. We discuss the implications of this below.

Estimating the total gas mass is more uncertain since it depends upon assumptions about the metallicity and on ionization corrections. Based on silicon, we estimate that the total mass of the gas traced by $\mathrm{Si}$ II, III, and IV in the outer CGM is $M_{\text {tot }}=0.57$ to $1.2 \times 10^{9} M_{\odot} Z_{\odot} / Z$, where $Z$ is the gas-phase silicon abundance in the CGM and $Z_{\odot}$ is its solar value. Werk et al. (2014) find a median metallicity of 0.2 solar in the CGM of COS-Halos galaxies, which implies $M_{\text {tot }} \sim 2.8$ to $6.3 \times 10^{9} M_{\odot}$ for the outer CGM in the COS-Burst sample. This is slightly lower than the mass estimated by Werk et al. for the inner CGM in typical galaxies.

\section{Discussion}

\subsection{Introduction}

We have found clear evidence that the outer CGM around the starbursts differs systematically compared to the control galaxies. The Ly $\alpha$, Si III, C IV, and possibly O VI absorption lines are stronger as a function of normalized impact parameter, and the ratios of the equivalent widths of Si III/Ly $\alpha$ and C IV/ Ly $\alpha$ are higher than in normal star-forming galaxies of the same stellar mass. Both the widths and the velocity offsets (relative to $v_{\text {sys }}$ ) of the absorption lines are also significantly larger in the starbursts than in the control galaxies. In fact, the implied velocities in the CGM of the COS-Burst galaxies are roughly twice the halo virial velocity, implying that some force other than gravity is affecting the dynamics. 

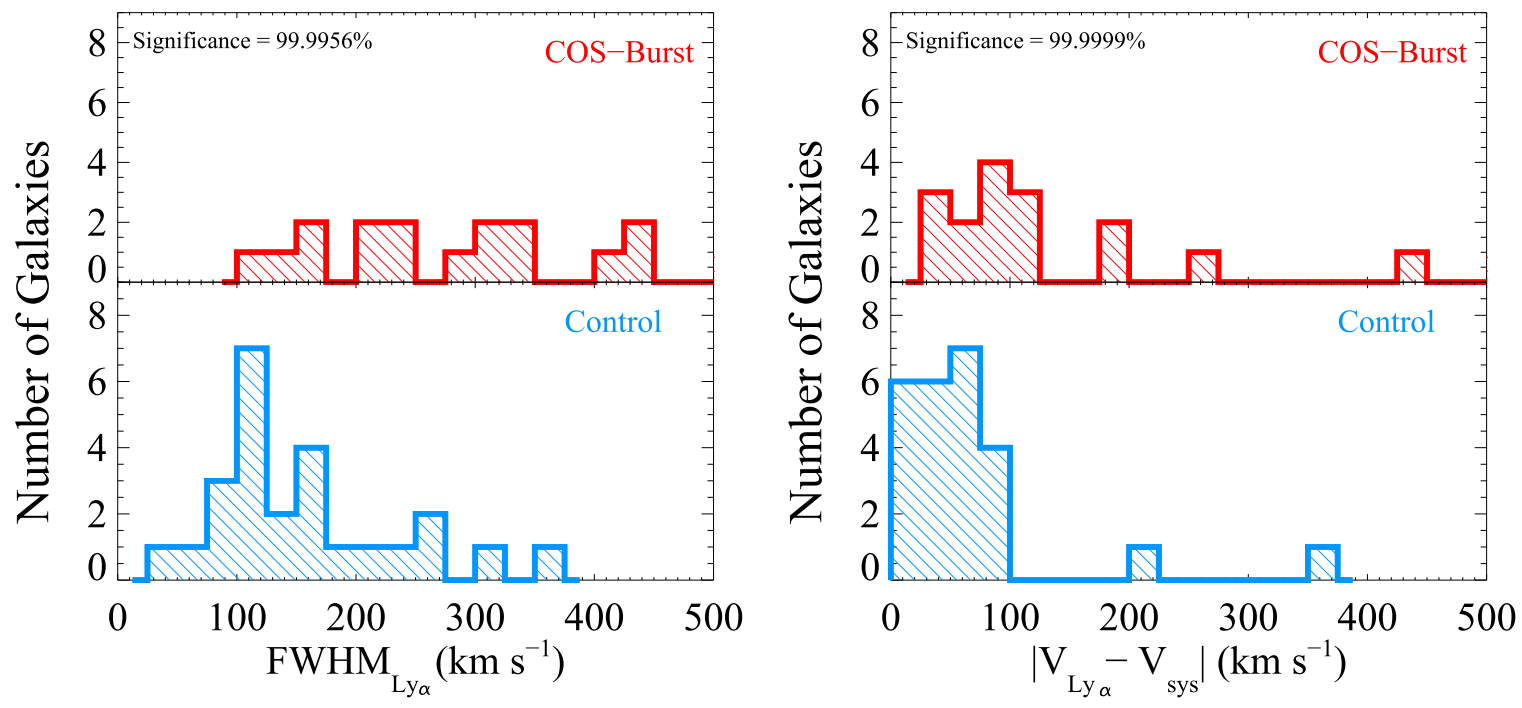

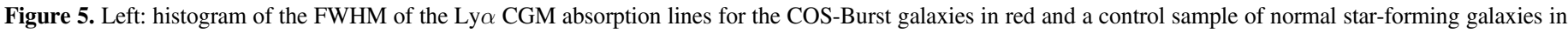

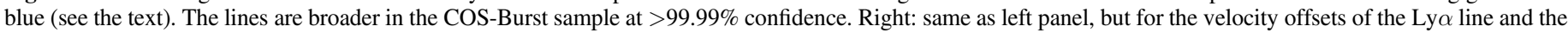
galaxy systemic velocity. The offsets are larger in the COS-Burst sample at the $99.99 \%$ confidence level.

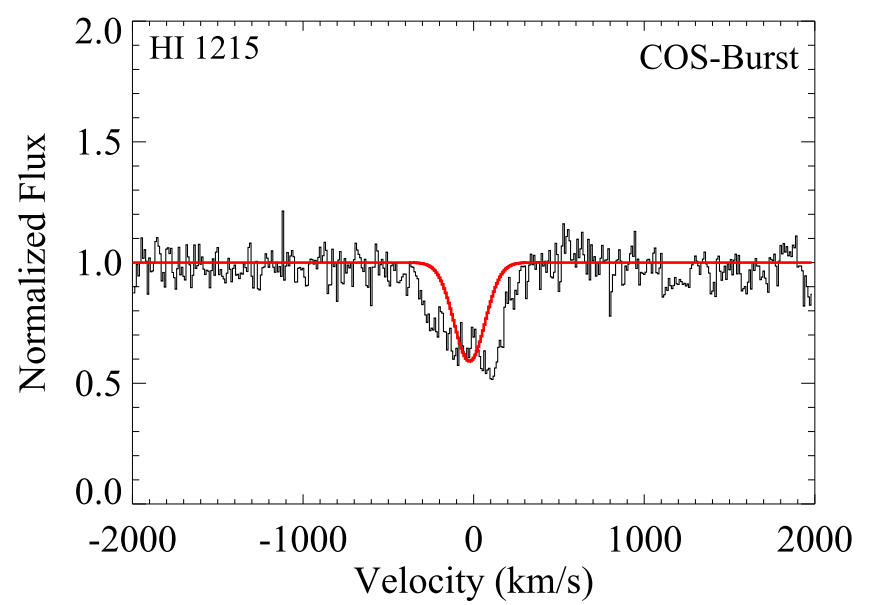

Figure 6. Stacked Ly $\alpha$ profile for the sample of 17 COS-Burst galaxies. The FWHM of this line is $424 \pm 25 \mathrm{~km} \mathrm{~s}^{-1}$. Overplotted in red is the profile expected for a population of absorbers moving randomly through the CGM at the median halo virial velocity for the sample. This profile has an FWHM of $214 \mathrm{~km} \mathrm{~s}^{-1}$ (only half of the observed value). Note that the asymmetric structure of the stacked profile reflects small number statistics in the stack.

It is important to recognize that the physical meaning of the line strength is not the same for all the lines. As noted above, the Ly $\alpha$ absorption lines are generally saturated, so the Ly $\alpha$ equivalent width is primarily tracing the spread in velocity of the absorbing gas along the line of sight (rather than column density). Thus, the stronger Ly $\alpha$ lines are connected to the different kinematic properties of the CGM in the COS-Burst galaxies noted above. In contrast, the Si III, C IV, and O VI lines have optical depths of roughly one. Their equivalent widths therefore trace the ionic column densities, and their detection fraction probes the covering factor of this gas in the CGM. The results above therefore mean that there are both more ionized metals and higher characteristic velocities in the outer CGM of the COS-Burst galaxies compared to the controls.

In the sections below, we consider various mechanisms that could link the starburst to the properties of the CGM.

\subsection{Alternatives to a Wind Model}

In the next section, we explore a model in some detail in which the CGM in our sample of COS-Burst galaxies is affected by a galactic wind that is driven by the starburst. Before doing so, we wish to consider possible alternative interpretations. First, we have shown that the CGM around the COS-Burst galaxies has unusual properties. What is the direction of the causal connection, however: is the starburst producing an unusual CGM, or is an unusual CGM fueling the starburst?

One possibility is that these starbursts have been triggered by major mergers that have affected the CGM that now surrounds the merger. This is not plausible in the case of the COS-Burst sample for a number of reasons. First, this idea would not explain the super-virial velocities we observe. Moreover, as can be seen in the imaging montage in Figure 2, the members of the COS-Burst sample are mostly normal late-type galaxies. A few appear to be interacting with companions, but few (if any) appear to be recent or on-going mergers. This is consistent with the fact that only about $12 \%$ of starbursts with SFRs like those of the COS-Burst sample are triggered by mergers (Sanders \& Mirabel 1996).

Another possibility is that the environment on the COSBurst galaxies differs systematically from that of normal starforming galaxies. For example, Johnson et al. (2015) found that there are differences in the CGM between isolated galaxies and galaxies in groups. To evaluate this, we have used the SDSS DR7 group catalog of Tago et al. (2010). This shows that 7 of the $17(41 \%)$ COS-Burst galaxies lie in groups. We have verified that there are no significant differences in the group versus isolated COS-Burst galaxies in Figures 4, 5, and 7 in this paper. Moreover, we find that about $60 \%$ of the control galaxies from COS-GASS are in groups.

A more general, and simple, way to consider whether the causal connection is an inward one is to ask whether it is plausible for the inflow or outflow of mass, metals, and energy to occur on the relevant timescale. The median value for the impact parameter in the sample is $\rho=179 \mathrm{kpc}$ and the median starburst age is $t_{\text {burst }} \sim 280$ Myr. A causal connection then 

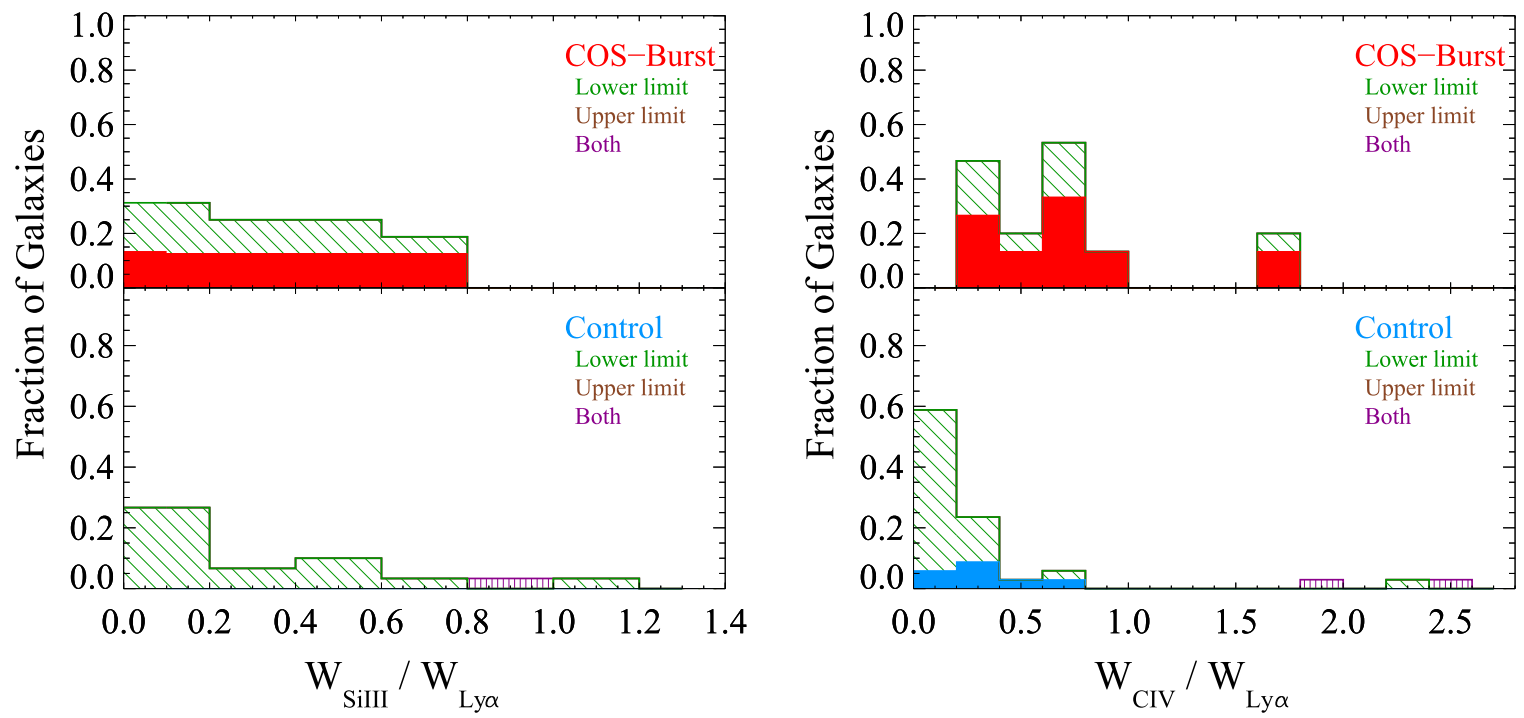

Figure 7. Histograms of the ratios of the Si III/Ly $\alpha$ and $\mathrm{C}$ IV/Ly $\alpha$ equivalent widths for the COS-Burst galaxies (top panels) and control star-forming galaxies (bottom panels). The cases with detections of the metal lines are shown in solid red (top) and solid blue (bottom). The green hatching shows the upper limits when the metal lines were not detected. Both ratios are higher in the COS-Burst sample. A Wilcoxon rank test shows that the median values are higher for the COS-Burst sample at the $95 \%$ (Si III) and $>99.99 \%$ (C IV) confidence level.

requires a characteristic velocity of $\rho / t_{\text {burst }}>630 \mathrm{~km} \mathrm{~s}^{-1}$. This represents the minimum velocity required for the outer part of the CGM to be causally connected to the starburst. This is considerably higher than the median halo circular velocities $\left(129 \mathrm{~km} \mathrm{~s}^{-1}\right)$. This makes it implausible that the causal connection is one in which the unusual properties of the outer CGM are related to the fueling of the starburst (e.g., an inward flow driven by gravity).

Of course, the innermost part of the CGM could be causally connected via inflow to the starburst. Observations of the inner CGM $(\rho<50 \mathrm{kpc})$ of star-forming galaxies at intermediate redshifts show enhanced $\mathrm{Mg}$ II absorption compared to quiescent galaxies, and the $\mathrm{Mg}$ II absorption is enhanced along the galaxy minor and major axis (Bordoloi et al. 2011; Bouché et al. 2012; Kacprzak et al. 2012; Ho et al. 2017). This may imply that a causal connection between the star formation and the CGM in both directions (outflow along the minor axis and inflow along the minor axis) may be occurring in the inner CGM. Unfortunately, we have only one sightline interior to $50 \mathrm{kpc}$, and none near the galaxy disk plane.

Returning to the outer CGM, the characteristic velocity required for causal connection can be more easily accommodated if the starburst is affecting this region (reflecting an outward flow). In this case, the flows of energy/mass/ metals at speeds well in excess of $v_{c}$ are possible (as we show below).

We now consider the effects of radiation from the starburst, which could very rapidly reach the outer CGM. We first consider the effects of ionizing radiation on the physical state of the CGM, and then the effects of non-ionizing UV radiation on the CGM dynamics. The CGM in normal galaxies is believed to be photoionized by the meta-galactic background (e.g., Werk et al. 2013, 2014). To assess whether the additional ionizing radiation from the starburst could be important, we can compare the relative intensities of these two sources. Following B13, this contribution from the starburst can be written as

$$
\Phi_{\mathrm{SB}}=1.4 \times 10^{5} \mathrm{SFR}_{100}^{-2} f_{\mathrm{esc}} \mathrm{cm}^{-2} \mathrm{~s}^{-1} .
$$

Here, the SFR is measured in units of $M_{\odot} \mathrm{yr}^{-1}$, the distance from the starburst to the CGM is measured in units of $100 \mathrm{kpc}$, and $f_{\text {esc }}$ is the fraction of ionizing photons that escape the starburst and reach the CGM. Using the median values for these parameters in Table 3 for our COS-Burst sample $\left(\mathrm{SFR}=12 \quad M_{\odot} \mathrm{yr}^{-1}, \quad r_{100}=1.79\right), \quad$ and the value $\Phi_{\mathrm{MGB}}=2750 \mathrm{~cm}^{-2} \mathrm{~s}^{-1}$ from Haardt \& Madau (2012), we find that ratio $\Phi_{\mathrm{SB}} / \Phi_{\mathrm{MGB}}=192 f_{\mathrm{esc}}$. In typical starbursts, there are only upper limits on $f_{\text {esc }}$ (one to a few percent-e.g., Heckman et al. 2011). ${ }^{8}$

While it is therefore possible that the starburst is contributing significantly to the photoionization of the CGM (for high enough values of $f_{\text {esc }}$, this would not naturally account for the stronger Si III we see in the outer CGM around the COS-Burst galaxies. As we have demonstrated above, the outer CGM in these galaxies contains a substantially higher mass of $\mathrm{Si}$ than is present there in normal galaxies. In both the COS-Burst and normal galaxies (Werk et al. 2014), the amount of Si III in the CGM is similar to or greater than the amounts of Si II and Si IV. Simply increasing the intensity of the ionizing radiation field in the CGM (thereby converting Si II to Si III and Si III to Si IV) cannot explain this difference in Si mass.

The CGM of the COS-Burst galaxies also differs from that of normal galaxies in terms of its kinematics (higher super-virial velocities). In principle, this could be the result of the acceleration of CGM clouds by the pressure of the far-UV radiation emitted

\footnotetext{
8 If the material in the outer CGM has been transported outward, the intensity of the ionizing radiation from the starburst would have been initially much higher (e.g., a factor of three smaller distance from the starburst would imply a radiation field nearly an order-of-magnitude larger). On the other hand, even if material near the starburst was initially photoionized by the starburst, the recombination times for the metal ions are so short that the material would recombine long before it reached the outer CGM. For the CGM cloud densities inferred for the inner CGM of $10^{-3} \mathrm{~cm}^{-3}$ (Werk et al. 2014), the recombination times for $\mathrm{C}$ IV to $\mathrm{C}$ III ( $\mathrm{Si}$ III to $\mathrm{Si}$ II) would only be about 4 (10) Myr (Nahar 1995; Nahar et al. 2000).
} 
by the starburst (e.g., Murray et al. 2005). Given the typical Si column densities in the CGM estimated above, and assuming a standard dust-to-metals ratio (Mattsson et al. 2014), the implied optical depth of the CGM clouds to far-UV radiation would only be of-order 0.002. Assuming an isothermal potential characterized by a velocity $v_{c}$, the ratio of the force due to radiation pressure to the force of gravity acting on a cloud is given by

$$
F_{\text {rad }} / F_{\text {grav }}=L \tau /\left(4 \pi c r v_{c}^{2} N_{H} \mu\right) .
$$

Here $L$ is the UV luminosity of the starburst, $\tau$ is the cloud dust optical depth, $r$ is the distance between the cloud and the starburst, $v_{c}$ is the halo circular velocity, $N_{\mathrm{H}}$ is the cloud hydrogen column density, and $\mu$ is the mean mass per particle $\left(1.4 m_{\mathrm{H}}\right)$ in the cloud. We use the parameters representing the medians in our sample $\left(L \sim 10^{43} \mathrm{erg} \mathrm{s}^{-1}, \tau=0.002\right.$, $\left.r=179 \mathrm{kpc}, \quad v_{c}=129 \mathrm{~km} \mathrm{~s}^{-1}\right)$. The column density of $\mathrm{Si}$ estimated above implies a total hydrogen column density of $N_{\mathrm{H}}=7 \times 10^{17}\left(Z_{\odot} / Z\right) \mathrm{cm}^{-2}$ (since the lower the metallicity, the higher the implied value of $N_{\mathrm{H}}$ for a given $N_{S i}$ ). We then find $F_{\text {rad }}=3.5 \times 10^{-4} Z / Z_{\odot} F_{\text {grav }}$. We conclude that radiation pressure will be dynamically negligible.

\subsection{A Galactic Wind}

We now assess the possible mechanisms by which a starburstdriven wind could affect the CGM. The physics of galactic winds driven by a population of massive stars has recently been reviewed by Heckman \& Thompson (2017). In addition to radiation pressure, the kinetic energy and momentum associated with stellar winds and supernova explosions play crucial dynamical roles. The stellar ejecta, through supersonic collisions, will create a hot volume-filling fluid inside the starburst (Chevalier \& Clegg 1985) with a temperature given by $T \sim 4 \times 10^{7} \mathrm{~K}(\alpha / \beta)$. Here $\alpha$ is the fraction of the kinetic energy injected by the massive stars that is not lost to radiative cooling, and $\beta$ is the ratio of the mass-injection rate to the SFR ( $\beta \sim 0.3$ corresponds to the pure stellar ejecta).

This hot gas will expand along the minor axis of the galaxy disk and blow out into the halo. The wind fluid will then cool adiabatically as it expands and will reach a terminal velocity given by $v_{\text {term }} \sim 1500 \mathrm{~km} \mathrm{~s}^{-1}(\alpha / \beta)^{1 / 2}$. In the discussion to follow, we take $\alpha=1$ and $\beta=0.3$, consistent with detailed modeling of the M82 wind (Strickland \& Heckman 2009). This implies $v_{\text {term }}=2800 \mathrm{~km} \mathrm{~s}^{-1}$. This velocity is high enough, in principle, for a wind to traverse the CGM on a timescale much shorter than the typical starburst lifetime.

One way to explain the differences between the CGM in the COS-Burst galaxies compared to normal star-forming galaxies is that a significant amount of metals have been transported to the outer CGM. Here we ask whether this is feasible based on the available energy delivered by a galactic wind. To set the stage, we note that for the median SFR and burst ages in our COS-Burst sample the implied kinetic energy released by supernovae and stellar winds will be $\sim 8 \times 10^{58} \mathrm{ergs}$ (Heckman \& Thompson 2017).

Above, we have estimated that the total gas mass in the outer CGM of the COS-Burst galaxies is about $5 \times 10^{9} M_{\odot}$. The work required to move this much mass by a factor of two in radius in an isothermal gravitational potential with $v_{c}=129 \mathrm{~km} \mathrm{~s}^{-1}$ would be about $10^{57} \mathrm{erg}$. This is almost two orders of magnitude smaller than the potentially available energy, so a starburst-driven wind could in principle rearrange the CGM. We now examine this model in more detail.

\subsubsection{A Wind-cloud Model}

The ram pressure of the wind fluid, combined with radiation pressure, will accelerate gas clouds and drive them outwards (e.g., Chevalier \& Clegg 1985; Murray et al. 2005). This process is believed to be responsible for the blueshifted interstellar absorption lines (seen in the down-the-barrel observations of starbursts) and the high-velocity optical emission-line gas seen on scales of $\sim 1-10 \mathrm{kpc}$ along the minor axes of starburst galaxies. These scales are far smaller than those we are probing here. Numerical simulations show that clouds accelerated in this way are unlikely to survive long enough to be transported over such large distances (see the discussion in Heckman \& Thompson 2017), so it is not plausible that clouds launched near the starburst could reach the outer CGM intact.

Instead, we consider a model in which we are observing the impact of the wind fluid on the preexisting clouds in the CGM, with properties like those derived by Werk et al. (2014). While the origin of these clouds is uncertain, the COS data provide very useful empirical information about their properties. We assume that the clouds have a relatively small volume-filling factor and that any diffuse volume-filling phase of the CGM can be ignored. In the next subsection we relax this assumption. To characterize the wind, we use parameters appropriate to the median values in our sample (Table 2).

As the hot wind fluid flows out into the CGM, its ram pressure can accelerate the preexisting CGM absorption line clouds it has overtaken. As in the case of radiation pressure considered above, we can compare the ram pressure and gravitational forces acting on a CGM cloud:

$$
F_{\text {ram }} / F_{\text {grav }}=\dot{p}_{\text {wind }} /\left(4 \pi r v_{c}^{2} N_{H} \mu\right) \text {. }
$$

Here $\dot{p}_{\text {wind }}$ is the momentum flux carried by the wind. For an SFR in $M_{\odot} \mathrm{yr}^{-1}$, this is given by

$$
\dot{p}_{\text {wind }}=1 \times 10^{34}(\alpha \beta)^{1 / 2} \text { SFR dynes. }
$$

Using the median values for these parameters in the COSBurst sample (Table 3), and taking $\alpha=1$ and $\beta=0.3$, we find $F_{\text {ram }} / F_{\text {grav }} \sim 34\left(Z / Z_{\odot}\right)$. Unless the metallicity in the CGM clouds is much lower than estimated by Werk et al. (2014) and Prochaska et al. (2017), the wind can overcome gravity and accelerate the clouds outward. This flow could in principle carry metals outward and account for the higher mass of metals in the outer CGM of the COS-Burst galaxies.

Heckman et al. (2015) derived the equation of motion for a cloud accelerated by the combined inward force of gravity and outward force due to a wind. In their notation, the ratio of the starburst momentum flux to the minimum needed to balance gravity is $R_{\text {crit }}=F_{\text {ram }} / F_{\text {grav }}$. They showed that the maximum velocity to which the cloud could be accelerated is given by

$$
v_{\max }=\sqrt{2} v_{c}\left[\left(R_{\text {crit }}-1\right)-\ln R_{\text {crit }}\right]^{1 / 2} .
$$

When we adopt $Z_{\mathrm{Si}} \sim 0.3 Z_{\odot}$ (Prochaska et al. 2017) to evaluate $R_{\text {crit }}$ (see above), this predicts a maximum outward velocity of $\sim 3.7 v_{c}$, or $\sim 480 \mathrm{~km} \mathrm{~s}^{-1}$ for the median value of $v_{c}=129 \mathrm{~km} \mathrm{~s}^{-1}$. For a median starburst lifetime of $280 \mathrm{Myr}$, a cloud moving at $480 \mathrm{~km} \mathrm{~s}^{-1}$ could travel a distance of about $135 \mathrm{kpc}\left(\sim 0.7 R_{\mathrm{vir}}\right)$. Thus, the large-scale transport of CGM clouds by this mechanism is at least potentially feasible.

To compare this outflow velocity to the width of observed stacked Ly $\alpha$ profile (Figure 6) requires a translation of the outflow velocity into a profile of the projected line-of-sight 
velocities. To do this, we have constructed a simple numerical model in which a spherically symmetric mass-conserving outflow travels at a constant velocity $v_{\text {out }}$, for a time sufficient to reach a maximum radial extent of $R_{\max }$. We have then measured the resulting FWHM along a line of sight through the outflow as a function of $\rho / R_{\max }$. We take $R_{\max } \sim 1.5 R_{\text {vir }}$ (the maximum value for $\rho$ in our sample). For the median value of $\rho / R_{\max }$ in our sample (0.63), the observed FWHM of $424 \mathrm{~km} \mathrm{~s}^{-1}$ implies $v_{\text {out }}=352 \mathrm{~km} \mathrm{~s}^{-1}$.

The wind will drive shocks into the clouds. Momentum balance across the shock implies that the shock velocity driven into a cloud initially at rest, by a wind flowing at $v_{\text {wind }}$, will be given by

$$
v_{\text {cloud }, s}=v_{\text {wind }}\left(n_{\text {wind }} / n_{\text {cloud }}\right)^{1 / 2} \text {. }
$$

Here, $n_{\text {cloud }}$ and $n_{\text {wind }}$ are the cloud and wind particle densities. Werk et al. (2014) find $n_{c} \sim 10^{-4} \mathrm{~cm}^{-3}$ in the outer CGM. Adopting the wind parameters as above, we calculate $n_{w} \sim 10^{-7} \mathrm{~cm}^{-3}$ at the radius matching the median impact parameter $(179 \mathrm{kpc})$. The implied value for $v_{\text {cloud,s }}$ would be about $10^{2} \mathrm{~km} \mathrm{~s}^{-1}$.

Could this shocked gas produce the observed Si III, C IV, and $\mathrm{O}$ VI absorption lines? First, we note that the radiative cooling time for the shocked gas will be $\sim 2\left(Z_{\odot} / Z\right) \mathrm{Myr}$, where $Z$ is the cloud metallicity. For the typical metallicities of $\sim 0.3$ solar inferred by Prochaska et al. (2017) and Werk et al. (2014), the cooling times are very short compared to the starburst lifetime. Shock models with velocities of $\sim 10^{2} \mathrm{~km} \mathrm{~s}^{-1}$ (e.g., Shull \& McKee 1979) can account for the observed ratios of the column densities of Si III, Si IV, and C IV. However, the ionic column densities of a single shock are small, and the observed values would imply that the line of sight through the CGM is intersecting tens of shocks. Shocks this slow do not produce significant O VI (e.g., Raymond 1979). If O VI in the SB/CGM has the same physical origin as the lower ions, a range in shock speeds would be required (e.g., from $\sim 100$ to $\sim 200 \mathrm{~km} \mathrm{~s}^{-1}$ ). A range of shock velocities is to be expected, since a range of cloud densities in the preexisting CGM is natural (Equation (6)).

An intriguing possibility is that the excess strength of the metal lines in the CGM of the COS-Burst galaxies could result from the destruction of CGM dust grains, releasing metals into the gas phase. This is a potentially important process: the amount of metals locked in dust grains in the CGM is similar to the amount present in the gas phase in typical star-forming galaxies (Ménard et al. 2010; Peeples et al. 2014). Calculations of grain destruction by shocks with speeds in the range we infer ( $\sim 100$ to $200 \mathrm{~km} \mathrm{~s}^{-1}$ ) show that significant fractions of Si and $\mathrm{C}$ that could be liberated, with the fraction increasing with increasing shock velocity and decreasing grain size (Draine \& Salpeter 1979 B. Draine 2017, private communication). While this possibility of dust destruction is not required in our model, it would have the advantage that the need for a bulk radial transport of gas-phase metals from the inner to outer CGM would not be required to explain all of the differences between the gas-phase metal distributions in the CGM of COS-Burst versus normal star-forming galaxies.

\subsubsection{A Two-phase CGM}

We now discuss how the above picture is modified when we add a diffuse volume-filling phase to the CGM. One immediate impact is that the timescale for the wind to affect the CGM can be significantly longer than in the case above. For simplicity, we consider a wind that propagates into a spherically symmetric volume-filling CGM. This will create an expanding wind-blown bubble, a general problem that has been analyzed by Castor et al. (1975), Dyson (1989) and Koo \& McKee (1992). From the inside out, the structure of the wind-blown bubble will be the starburst (where the energetic wind fluid is created), a sonic point, a region of freely streaming and adiabatically cooling supersonic wind-fluid, an internal (reverse) wind shock, thermalized (shock-heated) wind fluid, a contact discontinuity, shocked CGM material, and an external (outer) shock that is driven into the volume-filling CGM.

Observational constraints on the amount of hot volumefilling gas in the CGM of star-forming galaxies are rather poor. As summarized by Bland-Hawthorn \& Gerhard (2016) and Werk et al. (2014), there are estimates ranging from about $10^{9}$ to $10^{11} M_{\odot}$ for the halo of the Milky Way and other similar disk galaxies. The low densities (and hence low inferred pressures) in the CGM absorption line clouds found by Werk et al. (2014) for their sample would be more consistent with the low end of this range. We note that the median value for the stellar mass in our sample is $\sim 2.2 \times 10^{10} M_{\odot}$, only about $40 \%$ that of the Milky Way (Bland-Hawthorn \& Gerhard 2016). We therefore parameterize the properties of the hot volume-filling phase for our sample by adopting a fiducial value of $M_{\text {hot }}=10^{10} M_{\odot}$.

We have shown above that the amount of kinetic energy supplied by an energy-driven wind is nearly two orders of magnitude higher than what would be required to move $10^{10}$ $M_{\odot}$ of gas outward in the halo potential well. We therefore ignore gravity. The densities in the region of the shocked wind and shocked CGM are so low that the radiative cooling times will be much longer than a Hubble time. We therefore first consider an energy-driven bubble.

Both observations and theoretical models imply that the gas density in the CGM falls with radius roughly like $r^{-1}$ to $r^{-1.5}$ (Maller \& Bullock 2004; Miller \& Bregman 2013, 2015; Werk et al. 2014; Voit et al. 2017; Faermon et al. 2017). When we take an initial radial density profile for the volume-filling phase of $n_{v f} \propto 1 / r$, and following the self-similar solutions for energy-driven bubbles in Dyson (1989), the radius of the expanding bubble is given by

$$
r_{\text {bubble }}=86 \dot{E}_{43}^{1 / 4} M_{\mathrm{hot}, 10}^{-1 / 4} t_{8}^{3 / 4} \mathrm{kpc} .
$$

Here $\dot{E}_{43}$ is the rate of kinetic energy injected by the starburst in units of $10^{43} \mathrm{erg} \mathrm{s}^{-1}$ (corresponding to an SFR of $14 M_{\odot} \mathrm{yr}^{-1}$ ), $M_{\text {hot, } 10}$ is the total mass of the volume-filling phase out to a radius of $200 \mathrm{kpc}$ in units of $10^{10} M_{\odot}$, and $t_{8}$ is the time since the starburst began, given in units of $10^{8}$ years.

The median starburst age is $280 \mathrm{Myr}$ and the median kinetic energy injection rate implied by the median SFR is $9 \times 10^{42} \mathrm{erg} \mathrm{s}^{-1}$ for our COS-Burst sample. This leads to an outer radius for the expanding wind-blown bubble in the halo of $182 \mathrm{kpc}$. Once reaching this radius, the outer shock speed $\left(d r_{\text {bubble }} / d t\right)$ would be $480 \mathrm{~km} \mathrm{~s}^{-1}$.

We can also consider a momentum-driven bubble, allowing for the possibility that radiative cooling might be significant. In this case, the analysis in Dyson (1989) leads to

$$
r_{\text {bubble }}=99 \dot{p}_{35}^{1 / 3} M_{\text {hot }, 10}^{-1 / 3} t_{8}^{2 / 3} \text {. }
$$

The median momentum-flux for the wind in the COS-Burst sample would be $6.6 \times 10^{34}$ dynes for $\alpha=1$ and $\beta=0.3$ (see 
Equation (4) above). This implies then that $r_{\text {bubble }}=171 \mathrm{kpc}$, and the current outer shock speed is $\sim 400 \mathrm{~km} \mathrm{~s}^{-1}$. Thus, the momentum-driven case yields very similar values for the bubble size and expansion speed.

Given the highly idealized nature of our model and the uncertain mass of the volume-filling CGM, we regard these simple estimates as showing that it is plausible for a starburstdriven wind to affect the bulk of the CGM.

The interaction between this wind-driven bubble and preexisting clouds in the CGM will depend on the location of the cloud. It can initially be overtaken by the outer shock driven into the CGM, it can then be compressed in the region of the thermalized wind fluid. In the interior region occupied by the free wind, the wind can accelerate and shock clouds, as described in the previous section.

\subsubsection{Wind-stimulated Cloud Condensation}

In the model above, we have considered the interaction between a starburst-driven wind and preexisting clouds in the CGM. An interesting alternative is that the starburst-driven wind is instead actually creating the clouds seen in absorption by facilitating their condensation out of diffuse, thermally unstable gas in the CGM.

This idea has mostly been proposed and discussed in the context of the effects of AGN-driven outflows (jets) on the observed multiphase gas in the cores of clusters of galaxies $(\mathrm{Li}$ \& Bryan 2014; Voit et al. 2017). In this model, the AGNdriven outflow uplifts diffuse ambient gas. This uplifted gas cools adiabatically, thereby shortening its radiative cooling time and promoting the development of thermal instabilities and the formation of cold condensates (clouds). As noted by Voit et al., this idea could be generalized to the case of the CGM and outflows driven by feedback from massive stars.

In the context of this paper, this idea is attractive because it circumvents the difficulty of preventing wind-accelerated preexisting clouds in the CGM from being destroyed by hydrodynamical instabilities before they can be accelerated to high velocities or transported over significant distances (see Heckman \& Thompson 2017 and references therein).

\section{Conclusions}

The CGM represents the potential source of gas to fuel the future growth of the galaxy through accretion and subsequent star formation. In this paper we have investigated the effects of the energy and momentum released by a starburst on the CGM, in order to understand the role of such feedback in the evolution of galaxies. We have used Hubble Space Telescopes (HST) Cosmic Origins Spectrograph (COS) to measure the farUV spectra of background quasars along lines of sight passing within $\sim 230 \mathrm{kpc}$ of 17 low-redshift starburst and post-starburst galaxies (the COS-Burst sample). We have detected the CGM in absorption with Ly $\alpha$ in $100 \%$ of the possible cases, with Si III 1206.5 in $47 \%$, and with C IV 1548.2 in $44 \%$. In six cases we accessed the OVI 1031.9 line, detecting it in three cases (50\%). We have only upper limits for Si II 1260.4, Ci II 1334.5, and Si IV 1393.8.

We have used archival HST COS data to define control samples of normal star-forming galaxies selected to have the same range in stellar mass $\left(\sim 10^{10}-10^{11} M_{\odot}\right)$ and impact parameter as the COS-Burst sample. We then compared the properties of the CGM in the COS-Burst and control samples. We found the following results.

1. The Ly $\alpha$, Si III, and C IV absorption lines are significantly stronger in the CGM of the COS-Burst galaxies. This comparison was made as a function of normalized impact parameter $\left(\rho / R_{\text {vir }}\right)$. We note that this difference pertains to the outer $\operatorname{CGM}\left(\rho / R_{\text {vir }}>0.5\right)$, since we have few COS-Burst sightlines in the inner CGM.

2. Both the (nonparametric) FWHM and the velocity displacement of the individual $\operatorname{Ly} \alpha$ lines with respect to the galaxy systemic velocity $(\Delta v)$ are significantly larger for the COS-Burst galaxies. The stacked Ly $\alpha$ absorption line profile for the COS-Burst sample is roughly two times wider than the value expected if the clouds are moving through the CGM at the halo virial velocity $\left(\mathrm{FWHM}=424\right.$ versus $214 \mathrm{~km} \mathrm{~s}^{-1}$ ).

3. The ratios of the equivalent widths of the $\mathrm{Si}$ III and $\mathrm{C} \mathrm{IV}$ lines to those of Ly $\alpha$ are higher in the CGM of the COSBurst galaxies (by an average of $0.4 \mathrm{dex}$ ).

4. We conclude that the amount of metals is enhanced and the dynamical state of the (outer) CGM is significantly different in the COS-Burst sample.

The detected metal absorption lines are not usually saturated in the COS-Burst sample $(\langle\tau\rangle \sim 1)$. Using the measured impact parameters and column densities, we infer masses of $M_{\mathrm{Si} \text { II,III,IV }}=4.0$ to $10.0 \times 10^{5} M_{\odot}, M_{\mathrm{C} \text { IV }}=2.4 \times 10^{6} M_{\odot}$, and $M_{\mathrm{OVI}}=5 \times 10^{6} M_{\odot}$ for the outer CGM ( $\left.\rho=50-200 \mathrm{kpc}\right)$.

We next considered the causal relationship between the unusual properties of the CGM and the presence of a starburst. The COS-Burst galaxies are mostly normal late-type galaxies (not byproducts of recent major mergers). More generally, we argued that an inwardly directed connection (e.g., an unusual CGM leads to the triggering of a starburst) was unlikely. Given the typical starburst ages (median of $280 \mathrm{Myr}$ ) and impact parameters (median of $179 \mathrm{kpc}$ ), the required minimum velocity of the inflow would be $\sim 630 \mathrm{~km} \mathrm{~s}^{-1}$, compared to a median halo circular velocity of only $129 \mathrm{~km} \mathrm{~s}^{-1}$. We also explored the possibility that radiation pressure from the starburst could accelerate CGM clouds outward. Given the very low estimated dust optical depths of the clouds, this fails by many orders of magnitude.

We therefore considered the possible impact of a galactic wind driven by the collective momentum and/or energy supplied by supernovae and stellar winds in the starburst. We showed that (in the absence of a volume-filling CGM phase) a starburst-driven galactic wind could accelerate and shock-heat the types of clouds that are observed in the CGM of normal galaxies, and do so over the full range of observed impact parameters. We noted that these shocks might also liberate metals from CGM dust grains. If there is a significant volume-filling phase (as well as clouds), we showed that energy and/or momentum supplied by the starburstdriven wind could inflate a CGM-scale wind-blown bubble with the required size, on the required timescale. We also suggested that a starburst-driven wind could facilitate the creation of new clouds as adiabatically cooled uplifted diffuse gas in the CGM became thermally unstable.

While starbursts as strong as our sample are atypical in the present-day universe, they have specific star formation rates $\left(\sim 10^{-9} \mathrm{yr}^{-1}\right)$ that are typical of normal star-forming galaxies at 
redshifts of $\sim 0.5-3$, the epoch during which $\sim 80 \%$ of the present-day cosmic stellar inventory was created (Madau \& Dickinson 2014). Indeed, absorption line probes of the CGM around normal star-forming galaxies at $z \sim 2-3$ also show radial distributions of Ly $\alpha$ and C IV absorption lines extending out to impact parameters of $\sim 300$ and $\sim 100 \mathrm{kpc}$, respectively (Steidel at al. 2010). The physical picture we have proposed for the CGM of present-day starburst/post-starburst galaxies should be broadly relevant to the evolution of galaxies over cosmic time. Our data also yield new information about both starburst-driven winds and the CGM itself, as they show how the CGM responds to the injection of energy and momentum supplied by a wind on global scales. We believe that these new data can provide a valuable benchmark for future numerical simulations of the effects of feedback on galaxy evolution (e.g., Muratov et al. 2015; Simpson et al. 2015; Hopkins et al. 2017; Li et al. 2017).

This work is based on observations with the NASA/ESA Hubble Space Telescope, which is operated by the Association of Universities for Research in Astronomy, Inc., under NASA contract NAS5-26555. T.H. and S.B. were supported by grant HST GO 13862. V.W. acknowledges the support of the European Research Council via the award of a starting gant (SEDMorph: P.I. V. Wild).

This project also made use of archival SDSS data. Funding for the SDSS and SDSS-II has been provided by the Alfred P. Sloan Foundation, the Participating Institutions, the National Science Foundation, the U.S. Department of Energy, the National Aeronautics and Space Administration, the Japanese Monbukagakusho, the Max Planck Society, and the Higher Education Funding Council for England. The SDSS Web Site is http:// www.sdss.org/. The SDSS is managed by the Astrophysical Research Consortium for the Participating Institutions. The Participating Institutions are the American Museum of Natural History, Astrophysical Institute Potsdam, University of Basel, University of Cambridge, Case Western Reserve University, University of Chicago, Drexel University, Fermilab, the Institute for Advanced Study, the Japan Participation Group, Johns Hopkins University, the Joint Institute for Nuclear Astrophysics, the Kavli Institute for Particle Astrophysics and Cosmology, the Korean Scientist Group, the Chinese Academy of Sciences (LAMOST), Los Alamos National Laboratory, the Max PlanckInstitute for Astronomy (MPIA), the Max Planck-Institute for Astrophysics (MPA), New Mexico State University, Ohio State University, University of Pittsburgh, University of Portsmouth, Princeton University, the United States Naval Observatory, and the University of Washington.

T.H. thanks the Kavli Institute for Theoretical Physics, the Simons Foundation, and the Aspen Center for Physics for supporting informal workshops on galactic winds, feedback, and the circum-galactic medium during 2014, 2015, and 2016. These provided important input for this paper. Conversations at these workshops with Romeel Davé Crystal Martin, Norm Murray, Ralph Pudritz, Eliot Quataert, Brant Robertson, Chuck Steidel, Todd Thompson, and Ellen Zweibel were especially helpful. He also thanks Greg Bryan, Mark Voit, and Brian $\mathrm{O}$ 'Shea for interesting discussions of the possible importance of wind-driven precipitation.

Facility: Sloan (COS).

\section{ORCID iDs}

Timothy Heckman (ib https://orcid.org/0000-0001-6670-6370
Sanchayeeta Borthakur (D) https://orcid.org/0000-00022724-8298

\section{References}

Binney, J., \& Tremaine, S. 1987, Galactic Dynamics (Princeton, NJ: Princeton Univ. Press)

Bland-Hawthorn, J., \& Gerhard, O. 2016, ARA\&A, 54, 529

Bordoloi, R., Lilly, S., Knobel, L., et al. 2011, ApJ, 743, 10

Bordoloi, R., Lilly, S., Knobel, L., et al. 2014a, ApJ, 794, 130

Bordoloi, R., Tumlinson, J., Werk, J., et al. 2014b, ApJ, 794, 130

Borthakur, S., Heckman, T., Strickland, D., Wild, V., \& Schiminovich, D. 2013, ApJ, 768, 18

Borthakur, S., Heckman, T., Tumlinson, J., et al. 2015, ApJ, 813, 46

Borthakur, S., Heckman, T., Tumlinson, J., et al. 2016, ApJ, 833, 259

Bouché, N., Hohensee, W., Vargas, R., et al. 2012, MNRAS, 426, 801

Castor, J., McCray, R., \& Weaver, R. 1975, ApJ, 200, L107

Chevalier, R., \& Clegg, A. 1985, Natur, 317, 44

Chisholm, J., Tremonti, C., Calzetti, D., et al. 2015, ApJ, 811, 149

Draine, B., \& Salpeter, E. 1979, ApJ, 231, 438

Dyson, J. 1989, LNP, 350, 137D

Erb, D., Quider, A., Henry, A., \& Martin, C. 2012, ApJ, 759, 26

Faermon, Y., Sternberg, E., \& McKee, C. 2017, ApJ, 835, 52

Haardt, F., \& Madau, P. 2012, ApJ, 746, 125

Heckman, T., Alexandrof, R., Borthakur, S., et al. 2015, ApJ, 809, 147

Heckman, T., \& Borthakur, S. 2016, ApJ, 822, 9

Heckman, T., Borthakur, S., Overzier, R., et al. 2011, ApJ, 730, 5

Heckman, T., \& Thompson, T. 2017, Handbook of Supernovae (Berlin: Springer) in press

Ho, S., Martin, C. L., Kacprzak, G., \& Churchill, C. 2017, ApJ, 835, 267

Ho, T., Medling, A., Bland-Hawthorn, J., et al. 2016, MNRAS, 457, 1257

Hopkins, P., Wetzel, A., Keres, D., et al. 2017, arXiv:1702.06148

Johnson, S., Chen, H.-W., \& Mulchaey, J. 2015, MNRAS, 449, 3263

Kacprzak, G., Churchill, C., \& Nielsen, N. 2012, ApJ, 760, L7

Koo, B.-C., \& McKee, C. 1992, ApJ, 388, 93

Kornei, K., Shapley, A., Martin, C., et al. 2012, ApJ, 758, 135

Kravtsov, A., Vikhlinin, A., \& Meshscheryakov, A. 2014, arXiv:1401.7329

Li, M., Bryan, G., \& Ostriker, J. 2017, ApJ, 841, 101

Li, Y., \& Bryan, G. 2014, ApJ, 789, 153

Liang, C., \& Chen, H.-W. 2014, MNRAS, 445, 2061

Madau, P., \& Dickinson, M. 2014, ARA\&A, 52, 415

Maller, A., \& Bullock, J. 2004, MNRAS, 355, 649

Mandelbaum, R., Wang, W., Zu, Y., et al. 2016, MNRAS, 457, 3200

Martin, C., Shapley, A., Coil, A., et al. 2012, ApJ, 760, 127

Mattsson, L., De Cia, A., Anderson, A., et al. 2014, MNRAS, 440, 1562

Ménard, B., Scranton, R., Fukagita, M., et al. 2010, MNRAS, 405, 1025

Ménard, B., Wild, V., Nestor, D., et al. 2011, MNRAS, 417, 801

Miller, M., \& Bregman, J. 2013, ApJ, 770, 118

Miller, M., \& Bregman, J. 2015, ApJ, 800, 14

Muratov, A., Keres, D., Faucher-Gigure, C.-A., et al. 2015, MNRAS, 454, 2691

Murray, N., Quataert, E., \& Thompson, T. 2005, ApJ, 618, 569

Nahar, S. 1995, ApJS, 101, 423

Nahar, S., Pradhan, A., \& Zhang, H. L. 2000, ApJS, 131, 375

Peeples, M., Werk, J., Tumlinson, J., et al. 2014, ApJ, 786, 54

Prochaska, J. X., Kasen, D., \& Rubin, K. 2011, ApJ, 734, 24

Prochaska, J. X., Werk, J., Worseck, G., et al. 2017, ApJ, 837, 169

Raymond, J. 1979, ApJS, 39, 1

Rubin, K., Prochaska, J. X., Koo, D., et al. 2014, ApJ, 794, 156

Salim, S., Rich, R. M., Charlot, S., et al. 2007, ApJS, 173, 267

Sanders, D., \& Mirabel, F. 1996, ARA\&A, 34, 479

Shull, J. M., \& McKee, C. 1979, ApJ, 227, 131

Simpson, C., Bryan, G., Hummels, C., \& Ostriker, J. 2015, ApJ, 809, 69

Somerville, R., \& Davé, R. 2015, ARA\&A, 53, 51

Steidel, C., Erb, D., Shapley, A., et al. 2010, ApJ, 717, 289

Stocke, J., Keeney, B., Dansforth, C., et al. 2013, ApJ, 763, 148

Strickland, D., \& Heckman, T. 2009, ApJ, 797, 2030

Tago, E., Saar, E., Temple, E., et al. 2010, A\&A, 514, 102

Tumlinson, J., Thom, C., Werk, J., et al. 2011, Sci, 334, 948

Tumlinson, J., Thom, C., Werk, J., et al. 2013, ApJ, 777, 59

Voit, C. M., Meece, G., Li, Y., et al. 2017, ApJ, 845, 80

Weiner, B., Coil, A., Prochaska, J. X., et al. 2009, ApJ, 692, 187

Werk, J., Prochaska, J. X., Thom, C., et al. 2013, ApJS, 204, 17

Werk, J., Prochaska, J. X., Tumlinson, J., et al. 2014, ApJ, 792, 8

Wild, V., Charlot, S., \& Heckman, T. 2010, MNRAS, 405, 933

Wild, V., Kauffmann, G., Heckman, T., et al. 2007, MNRAS, 381, 543 